\title{
An investigation of how radiation may cause accelerated rates of tropical cyclogenesis and diurnal cycles of convective activity
}

\author{
M. E. Nicholls \\ University of Colorado, Department of Atmospheric and Oceanic Sciences, Cooperative Institute for Research in \\ Environmental Sciences, Boulder, CO, USA
}

Correspondence to: M. E. Nicholls (melville.nicholls@ colorado.edu)

Received: 17 August 2014 - Published in Atmos. Chem. Phys. Discuss.: 4 March 2015

Revised: 27 May 2015 - Accepted: 18 July 2015 - Published: 13 August 2015

\begin{abstract}
Recent cloud-resolving numerical modeling results suggest that radiative forcing causes accelerated rates of tropical cyclogenesis and early intensification. Furthermore, observational studies of tropical cyclones have found that oscillations of the cloud canopy areal extent often occur that are clearly related to the solar diurnal cycle. A theory is put forward to explain these findings. The primary mechanism that seems responsible can be considered a refinement of the mechanism proposed by Gray and Jacobson (1977) to explain diurnal variations of oceanic tropical deep cumulus convection. It is hypothesized that differential radiative cooling or heating between a relatively cloud-free environment and a developing tropical disturbance generates circulations that can have very significant influences on convective activity in the core of the system. It is further suggested that there are benefits to understanding this mechanism by viewing it in terms of the lateral propagation of thermally driven gravity wave circulations, also known as buoyancy bores. Numerical model experiments indicate that mean environmental radiative cooling outside the cloud system is playing an important role in causing a significant horizontal differential radiative forcing and accelerating the rate of tropical cyclogenesis. As an expansive stratiform cloud layer forms aloft within a developing system the mean low-level radiative cooling is reduced, while at mid levels small warming occurs. During the daytime there is not a very large differential radiative forcing between the environment and the cloud system, but at nighttime when there is strong radiative clear-sky cooling of the environment it becomes significant. Thermally driven circulations develop, characterized by relatively weak subsidence in the environment but much stronger upward motion in the cloud system. This upward motion leads to a cooling ten-
\end{abstract}

dency and increased relative humidity. The increased relative humidity at night appears to be a major factor in enhancing convective activity, thereby leading in the mean to an increased rate of genesis. It is postulated that the increased upward motion and relative humidity that occur throughout a deep layer aid both in the triggering of convection and in providing a more favorable local environment at mid levels for maintenance of buoyancy in convective cells due to a reduction of the detrimental effects of dry air entrainment. Additionally, the day/night modulations of the environmental radiative forcing appear to play a major role in the diurnal cycles of convective activity in the cloud system. It is shown that the upward velocity tendencies in the system core produced by the radiative forcing are extremely weak when compared to those produced by latent heat release in convective towers, but nevertheless over the course of a night they appear capable of significantly influencing convective activity.

\section{Introduction}

Numerous studies utilizing IR satellite imagery have shown that there is a significant diurnal cycle of cirrus cloud cover in tropical cyclones (e.g., Browner et al., 1977; Muramatsu, 1983; Lajoie and Butterworth, 1984; Steranka et al., 1984; Kossin, 2002). The maximum areal extent of cloud canopies was found to occur in the early morning and the minimum in the early evening. It has been generally thought that the cause is a diurnal oscillation in deep convection near the storm center (Hobgood, 1986). Browner et al. (1977) suggested that the oscillation should also be associated with a diurnal cycle of 
rainfall. A recent study by Shu et al. (2013) has confirmed this supposition, showing that a significant diurnal variation of rainfall occurs in western North Pacific tropical cyclones.

Recent numerical modeling studies also suggest that radiation may increase the rate of tropical cyclogenesis (Nicholls and Montgomery, 2013, hereafter NM13; Melhauser and Zhang, 2014). Studies previous to these, although limited by relatively coarse grid resolution and parameterization of moist convection, had already shown earlier intensification when a simple longwave cooling scheme was included (Sundqvist, 1970; Hack and Schubert, 1980). NM13 conducted idealized experiments of tropical cyclogenesis using the Regional Atmospheric Modeling System (RAMS) developed at Colorado State University (Pielke et al., 1992; Cotton et al., 2003). The objective was to obtain a better understanding of two distinctly different pathways to tropical cyclogenesis that occurred in the idealized numerical modeling studies of Montgomery et al. (2006) and Nolan (2007). The latter two investigations examined the transformation of a relatively weak initial vortex over a warm ocean surface into a tropical cyclone using grid resolutions marginally capable of resolving convective clouds. Interestingly, the results of the two studies were very different. Montgomery et al. (2006) conducted simulations with RAMS and found a pathway occurring similar to that in a case study of the formation of Hurricane Diana (1984) by Hendricks et al. (2004). Vortical hot towers (VHTs) were the preferred coherent structures. The aggregate diabatic heating of the VHTs provided an influx of low-level angular momentum, causing low-level cyclonic winds to increase. Eventually the low-level winds became stronger than those of the mid-level vortex used to initialize the model and remained stronger or comparable to the winds aloft until they reached tropical depression strength (considered by NM13 to be approximately $12 \mathrm{~m} \mathrm{~s}^{-1}$ ). Development was characterized by a gradual decrease in the radius of maximum winds. Vorticity gradually built in the center as the system-scale inflow produced increasing cyclonic vorticity and as small-scale cyclonic vorticity anomalies were converged at low levels and underwent aggregation. In contrast the simulations by Nolan (2007) with the Weather Research and Forecasting (WRF) model showed a gradual strengthening of the initial mid-level vortex followed by the sudden formation of a small surface concentrated vortex near the center of the larger-scale circulation, with a radius of maximum winds of only a few kilometers. This vortex became the focus of a strengthening cyclonic circulation that grew in size to form a small tropical cyclone. These differing results were perplexing since the two pathways were distinctly different even though there were many similarities between the models and the experimental designs.

Nicholls and Montgomery (2013), using a newer version of RAMS, were able to demonstrate for the first time development along both pathways, depending on initial conditions and the model physics employed. There were some caveats to the development along the second pathway. Instead of the initial mid-level vortex simply strengthening, the surface cyclonic winds typically increased to begin with, similarly to the first pathway. It was only when an extensive stratiform anvil had formed aloft that a second prominent mid-level vortex developed. NM13 ran a set of experiments to investigate the two pathways described by Montgomery et al. (2006) and Nolan (2007) in more detail, which were denoted pathway One and pathway Two, respectively. They concluded that the ice phase was crucial for the formation of a strong mid-level vortex and development along pathway Two. Environments conducive to forming large quantities of ice aloft appeared to be more favorable for development along pathway Two. Higher sea surface temperatures for instance appeared to produce more intense and deeper convective cells, and more ice aloft, and therefore favored evolution along the second pathway.

NM13 included simulations both with and without radiation, and comparison of the genesis rate for otherwise identical experiments reveals significant differences. Table 1 shows results for four pairs of experiments that are exactly the same except for whether radiation is included or not. The time that maximum azimuthally averaged tangential winds reach $12 \mathrm{~m} \mathrm{~s}^{-1}$ is much quicker for the experiments with radiation included for each of the four pairs. The subsequent time to go from winds of $12 \mathrm{~m} \mathrm{~s}^{-1}$ to tropical storm strength winds of $17.4 \mathrm{~m} \mathrm{~s}^{-1}$ is also considerably faster for all cases. On the other hand, there is no systematic increase in the later intensification rate from tropical storm strength to hurricane strength $\left(33 \mathrm{~m} \mathrm{~s}^{-1}\right)$ when radiation is included. NM13 also found a strong diurnal cycle of convective activity when radiation was included.

Potential influences of radiation on oceanic tropical mesoscale convective systems (MCSs) and tropical cyclones include enhancing surface precipitation, causing diurnal cycles, changing the rate of development and effecting structure and motion. There are three main mechanisms that have been proposed for the role of radiation in these convective systems: (1) differential cooling between the weather system and its surrounding cloud-free region (Gray and Jacobson, 1977); (2) large-scale clear-sky environmental cooling (Dudhia, 1989; Tao et al., 1996); (3) changing thermal stratification due to cloud top and cloud base radiative forcing (Webster and Stephens, 1980; Hobgood, 1986; Xu and Randall, 1995).

The first mechanism was proposed by Gray and Jacobson (1977), who presented observational evidence in support of the existence of a large diurnal cycle of oceanic, tropical deep cumulus convection. They found that in many places, heavy rainfall is 2 to 3 times greater in the morning than in the late afternoon and evening. They hypothesized that the clear environment surrounding the weather system experiences significant deep radiative cooling during the nighttime, whereas the air within the cloud system experiences little radiative forcing except near the cloud top, where there is strong cooling. They propose that the environmental cool- 
Table 1. General statistics comparing four cases without and with radiation from NM13. Moist, dry refers to the core having an initial moisture anomaly or not. All cases are for a weak initial vortex having a maximum tangential wind speed of $8 \mathrm{~m} \mathrm{~s}^{-1}$ at $z=4 \mathrm{~km}$. Shown are the time the maximum averaged tangential wind speeds at the surface reach $12 \mathrm{~m} \mathrm{~s}^{-1}$; the near-surface radius of maximum winds (RMW) at this time; the pathway taken to genesis; the time at which the system becomes a tropical storm (TS); the RMW at this time; the time the system becomes a hurricane $(\mathrm{H})$; and the RMW at this time.

\begin{tabular}{|c|c|c|c|c|c|c|c|c|}
\hline Exp. & Description & $T_{12}(\mathrm{~h})$ & $\begin{array}{l}\mathrm{RMW}_{12} \\
\quad(\mathrm{~km})\end{array}$ & Path & $T_{\mathrm{TS}}(\mathrm{h})$ & $\begin{array}{l}\mathrm{RMW}_{\mathrm{TS}} \\
\quad(\mathrm{km})\end{array}$ & $T_{\mathrm{H}}(\mathrm{h})$ & $\begin{array}{c}\mathrm{RMW}_{\mathrm{H}} \\
(\mathrm{km})\end{array}$ \\
\hline 1 & $\begin{array}{l}\text { No radiation, moist, small, } \\
\text { weak, SST } 29\end{array}$ & 82 & 9 & 2 & 92 & 13 & 103 & 13 \\
\hline 2 & $\begin{array}{l}\text { Radiation, moist, small, weak, } \\
\text { SST29 }\end{array}$ & 60 & 5 & 2 & 65 & 11 & 78 & 13 \\
\hline 3 & $\begin{array}{l}\text { No radiation, dry, small, weak, } \\
\text { SST29 }\end{array}$ & 151 & 5 & 2 & 174 & 11 & 189 & 13 \\
\hline 4 & $\begin{array}{l}\text { Radiation, dry, small, weak, } \\
\text { SST29 }\end{array}$ & 101 & 11 & 2 & 105 & 13 & 119 & 15 \\
\hline 5 & $\begin{array}{l}\text { No radiation, moist, large, } \\
\text { weak, SST29 }\end{array}$ & 89 & 5 & 2 & 103 & 23 & 114 & 23 \\
\hline 6 & $\begin{array}{l}\text { Radiation, moist, large, weak, } \\
\text { SST29 }\end{array}$ & 55 & 5 & 2 & 61 & 19 & 69 & 15 \\
\hline 7 & $\begin{array}{l}\text { No radiation, moist, large, } \\
\text { weak, SST28 }\end{array}$ & 112 & 41 & 1 & 118 & 37 & 127 & 23 \\
\hline 8 & $\begin{array}{l}\text { Radiation, moist, large, weak, } \\
\text { SST28 }\end{array}$ & 49 & 5 & 2 & 52 & 9 & 68 & 15 \\
\hline
\end{tabular}

ing causes increased subsidence and low-level convergence into the cloud cluster at night, which enhances convection during the morning. During the daytime the solar heating of the environment offsets the longwave cooling to a large extent and environmental subsidence and low-level convergence into the cloud cluster are reduced, thereby producing a diurnal cycle of convective intensity. They emphasize that it is the larger low-level moisture convergence that occurs in the morning that is responsible for the higher morning precipitation rates. Several numerical modeling studies have investigated the potential of this differential radiation forcing mechanism to cause diurnal cycles of convective intensity in MCSs and conclude that it is probably of relatively minor importance (Dudhia, 1989; Miller and Frank, 1993; Xu and Randall, 1995; Tao et al., 1996). All of these studies were in two dimensions; further details will be provided in the discussion of the other two mechanisms. There have not been many numerical modeling studies that have investigated the importance of the horizontal differential heating mechanism in tropical cyclones. Craig (1996), utilizing an axisymmetric model with explicit convection, examined the effect of radiation for an initial vortex with maximum winds of $15 \mathrm{~m} \mathrm{~s}^{-1}$ at a radius of $75 \mathrm{~km}$. Development was quite rapid with azimuthal winds reaching approximately $60 \mathrm{~m} \mathrm{~s}^{-1}$ by $45 \mathrm{~h}$. There was little difference between simulations with and without radiation until $60 \mathrm{~h}$. The longwave cooling was found to increase the maximum intensity by about $20 \%$. Results of sensitivity experiments suggested that differential cooling was the sole mechanism responsible for the enhanced deepening. It was noted that the lack of difference in the early development could have been due to the cloud pattern not being established until $40-50 \mathrm{~h}$, implying little contrast between cloud and cloud-free regions. Also, it was pointed out that initialization with an initial vortex of $15 \mathrm{~m} \mathrm{~s}^{-1}$ without cloud is extreme, since in nature the cloud is likely to precede the establishment of a balanced vortex. On the other hand, it was noted that the cloud distribution early in the development of a tropical cyclone is likely to be highly variable. Results of the study were not considered conclusive and further work in this area was recommended.

Some modeling studies support the idea that large-scale clear-sky environmental cooling can increase precipitation rates in MCSs. Dudhia (1989) used a two-dimensional hydrostatic model with parameterized convection to investigate the life cycle of an MCS in the South China Sea that developed near the coast of Borneo. The MCS was a slow moving system with convective cores that were embedded mainly on the upwind side of a broad area of stratiform precipitating cloud. Sensitivity tests indicated that radiative clear-sky cooling aided the convection by continually destabilizing the troposphere. Two-dimensional numerical modeling studies by Miller and Frank (1993) and Fu et al. (1995) of MCSs in an environment typical of the eastern Atlantic Intertropical Convergence Zone also emphasized the importance of large-scale clear-sky cooling. Both of these studies simulated cloud lines with trailing cloud anvils. Miller and Frank (1993) examined the sensitivity to removing the horizontal radiative gradients, while retaining domain-wide radiative destabilization. They found that this resulted in only a small difference in rainfall, leading them to conclude that large-scale radiative destabi- 
lization was the main factor causing enhanced rainfall rates when radiation was included. Tao et al. (1996) used a twodimensional non-hydrostatic cloud-resolving model to simulate the development of both a tropical oceanic squall line and a mid-latitude continental squall line. Again, this study found that large-scale clear-sky radiative cooling played an important role. However, their experiments indicated that it was not so much destabilization that enhanced surface rainfall in their simulations when longwave radiation was included, but increased relative humidity. They found that convective available potential energy (CAPE) was not significantly increased by large-scale clear-sky cooling. They emphasize that increased relative humidity due to cooling allows condensation to occur more readily. Furthermore, it reduces evaporation and the negative impact of dry air entrainment. These are important additional insights into how large-scale clear-sky radiative cooling works in this context. Tao (1996) also found that solar heating reduced precipitation compared with runs with longwave forcing only, and suggested that this was likely to be playing a significant role in the diurnal precipitation cycle found over most oceans.

Tao et al. (1996) calculated time- and domain-averaged longwave radiative profiles over the clear and cloudy regions for both squall lines that were simulated (Fig. 13 of their manuscript). For the tropical oceanic case below $11 \mathrm{~km}$, the clear-sky longwave cooling was approximately $1.5 \mathrm{~K} \mathrm{day}^{-1}$ larger than for the cloudy regions. This is substantial, and it will be shown in this paper that such a difference, when operative for a 12-h period, should produce an unbalanced overturning circulation with significant consequences for convection. As a sensitivity experiment they eliminated differential cooling between cloudy and cloud-free regions by replacing the cloudy heating/cooling profiles with cloud-free radiative cooling. They found that for both the tropical and midlatitude cases surface rainfall was actually increased, which led them to conclude tentatively that differential cooling was not the mechanism responsible for enhancing the surface precipitation when longwave radiation was activated in the model. An additional sensitivity test was run in which longwave cooling was allowed to act for $6 \mathrm{~h}$ prior to triggering convection, and then the simulation was run without any radiative processes. For the tropical oceanic case, which had a small saturation deficit, there was a significant increase in surface rainfall similar to that occurring for the simulation with full radiative interactions. These experiments led them to conclude that the increase in humidity due to large-scale radiative cooling was significantly more important than the convergence/differential radiation mechanism ${ }^{1}$ (Gray and Jacobson, 1977).

There are several important caveats to this large-scale cooling mechanism that are important for understanding its potential influence on tropical cyclones and their simulation. Typically model simulations are initialized without the initial presence of cloudy air and it takes several hours, often 6-12 h, before deep convection develops. During this period, large-scale radiative cooling can occur, the amount of course being influenced by what time of day the model is initialized, which results in increased relative humidity and possibly changes in stability that can enhance convective activity. Once a thick stratiform anvil forms aloft, which is the result of the merging of anvils from numerous deep convective cells, clear-sky cooling becomes confined to the surrounding relatively cloud-free environment. At this point there are two potentially important effects: the surrounding environment becomes more conducive to the development of widespread convection as cloud-free cooling continues in this region, and the low-level inflow of air into the system from the environment becomes more humid, compared to a simulation without radiation. It has yet to be shown how important these effects might be during the early stage of a tropical cyclone's lifetime. Moreover, the question of whether day-night modulations of the radiative forcing in the surrounding environment can by these processes cause the diurnal variations observed in tropical cyclones has yet to be ascertained.

Another caveat to the large-scale radiative cooling mechanism is that it can take place in between and above scattered cumulus and cumulus congestus. Also, large-scale radiative cooling above a low-level stratocumulus layer can lead to increases in humidity and stability changes aloft. In this situation large-scale cooling would be a more appropriate term than clear-sky cooling for causing the increased potential for convective activity.

The third mechanism mentioned is based on the finding that large values of radiative heating during the day and cooling at night occur at the top of oceanic tropical cloud canopies, as well as large longwave radiative heating at cloud base (Webster and Stephens, 1980). The authors of this study concluded that there could be substantial destabilization of

\footnotetext{
${ }^{1}$ The distinction between this mechanism and the first, even though the first also involves clear-sky radiative cooling, is that the resultant cooling of the air and increase in relative humidity occurs in situ, where the clear-sky radiative cooling occurs, and is not a result of radiatively induced circulations. When radiative cooling is horizontally homogeneous and there are no clouds, what happens is clear: the air will cool and there will be an increase in relative humidity in situ. However, when there are clouds present, radiatively induced circulations can occur, causing subsidence in the clear-sky region and an associated adiabatic warming tendency that offsets the radiative cooling in a subsiding air parcel. At a particular level drying may occur due to subsidence, since the air aloft is usually drier, leading to reduced relative humidity. In this situation these effects need to be considered as well.
} 
the cloud layer due to this radiative forcing, particularly at night. Xu and Randall (1994) refer to this mechanism as "direct radiation-convection interaction", and results of their ensemble numerical model simulations led them to conclude that it plays a dominant role in diurnal cycles of deep convection over the tropical oceans. On the other hand, some of the studies that have focused on individual MCSs suggest this mechanism is not as important as large-scale clear-sky cooling in enhancing surface precipitation (Miller and Frank, 1992; Fu et al., 1995; Tao et al., 1996).

This third mechanism has also been by examined in regard to tropical cyclones by Hobgood (1986), who suggested that variations in thermal stratification aloft due to cloud top cooling at night and warming during the daytime might possibly cause the observed diurnal oscillations of the areal extent of cloud canopies. Numerical modeling results presented by Hobgood (1986) indicated that the diurnal cycle of net radiation at the cloud tops was the primary cause of the oscillations. Radiative cooling at night steepens the lapse rate and increases convection. During daylight hours, the absorption of solar radiation reduces the lapse rate, thus resulting in a minimum in convection. It was also suggested that this process might be augmented by differential cooling of cloudy and clear areas, as proposed by Gray and Jacobson (1977). While this early numerical model provided strong evidence of diurnal oscillations in convective activity in tropical cyclones, it was very basic compared to today's models and was unable to actually reproduce diurnal oscillations of the cirrus canopy.

A numerical modeling study of the effect of the diurnal radiation cycle on the pre-genesis environment of Hurricane Karl (2010) using the Advanced Research WRF has been reported recently by Melhauser and Zhang (2014). An observational analysis by Davis and Ahijevych (2012) found an approximate diurnal cycle of convective fluctuations with a maximum in the mid to late morning and a minimum in the late evening leading up to genesis of Karl. The numerical modeling sensitivity tests showed a case where inclusion of both shortwave and longwave components of radiation led to genesis and intensification, whereas a simulation without radiation did not develop. Furthermore, it was found that a simulation with nighttime-only radiation had a fast genesis and intensification, whereas a daytime-only radiation case did not develop. Therefore, these results indicate an important role of radiation in increasing the genesis rate, in agreement with NM13, and also showed significant day-night differences of radiation on TC development. The effects of radiation in the Melhauser and Zhang (2014) study were analyzed in terms of the "local environment" by horizontally averaging each model level within a circle of radius $225 \mathrm{~km}$ from the vortex center and the "large scale environment" by averaging over an annulus from 300 to $450 \mathrm{~km}$. The effects of radiation were then independently assessed in each region. Their presented results did not explicitly illustrate diurnal cycles of convective activity. The focus, rather, was on the simulated early development. They noted that their results appeared consistent with the conclusions of previous studies regarding destabilization due to large-scale environmental cooling, particularly at night, by Dudhia (1989), Miller and Frank (1993), and Tao et al. (1996). During the daytime they conclude that local and large-scale reduction of relative humidity and increased stability made the overall environment less conducive to deep moist convection. Their study apparently did not examine any potential role of horizontal differential radiative forcing in producing diurnal oscillations of convective activity.

Another recent numerical modeling study by $\mathrm{Bu}$ et al. (2014) investigated the influence of cloud-radiative forcing on tropical cyclones. The primary model they used was Hurricane WRF. The initialization that they employed resulted in winds of tropical storm strength being reached by $24 \mathrm{~h}$, so they did not focus on the early stages of development. The impact of cloud-radiative forcing was examined by comparing the development of a tropical cyclone having only clear-sky radiation with one having both clear-sky and cloud radiation. The main impact of including cloudradiative forcing at this stage was to significantly broaden the wind field. A similarity was noted between their results for the vertical profiles of diurnally averaged net radiative forcing for clear and disturbed (cloudy) regions and those presented by Gray and Jacobson (1977). Sensitivity tests showed that weak, primarily longwave, warming within the cloud anvil was the major factor responsible for modifying the structure when cloud-radiative forcing was included.

The present study examines the influence of radiative forcing on tropical cyclogenesis, early intensification, and diurnal oscillations of convective activity. As already discussed there appear to be limitations in the ability of the largescale environmental cooling mechanism to explain continual diurnal cycles in a developing tropical cyclone once a cloud shield has formed, and also to explain the increased genesis rate observed in recent numerical simulations. Increased relative humidity of the inflow air during the nighttime could possibly explain the cycles of precipitation rate. On the other hand, increasing environmental relative humidity at night could cause more widespread convection possibly more conducive to widening the low-level circulation rather than strengthening it. This mechanism may indeed be important, but it is not clear that it is the primary factor. The third mechanism also is problematic for explaining an increased genesis rate. The very large oscillations of radiative forcing at cloud top between the night and day could certainly influence ice growth and the strength of convection aloft. It is not clear however that this could lead to an increased low-level inflow at night or a mean increase in low-level convergence that could enhance the genesis rate. The first mechanism discussed has been found to be of minor importance in several numerical modeling studies of MCSs; nevertheless, simple idealized experiments that will be presented in this paper suggest that it should be capable of raising relative humidity 
values considerably during the nighttime in the cloud system throughout a deep layer. Therefore, it is proposed here that this mechanism is likely to be playing a significant role in both increasing the genesis rate and causing diurnal cycles of convective activity.

This is a preliminary study, but makes a strong case for the mechanism proposed by Gray and Jacobson (1977) to explain diurnal cycles of precipitation rate in MCSs as being relevant for tropical cyclones in their early stages of development. Furthermore, it is beneficial to adopt a dynamical perspective recognizing that modulations of the horizontal differential radiative heating generate thermally forced gravity waves, also known as buoyancy bores (Nicholls et al., 1991; Mapes, 1993). Early work that examined the transient linear response of a stratified atmosphere to prescribed heat sources as a simple model of moist convection noted that thermally generated gravity waves were playing a fundamental role in compensating subsidence (Lin and Smith, 1986; Raymond, 1986; Bretherton and Smolarkiewicz, 1989). Following these works Nicholls et al. (1991) derived simple analytical twodimensional solutions to the linear hydrostatic Boussinesq equations for an atmosphere at rest with prescribed heat sources and sinks. For a case with an idealized rigid lid and a deep heat source, represented by a half-sine wave in the vertical, the thermally generated buoyancy circulation was characterized by upward motion in the heated region with outflow aloft and inflow at low levels. The outflow and inflow expanded rapidly on either side of the heat source. The leading edges of these expanding circulations were deep fast moving wave-like pulses of subsidence. So, the subsidence compensating the central upward motion did not occur continually over broad regions on either side of the heat source, but had a distinct horizontally propagating character. The propagating subsidence regions caused adiabatic warming, adjusting the environmental potential temperature towards the perturbed values at the heated center for this two-dimensional framework with a rigid lid. Response to a thermal forcing profile more typical of an MCS having a stratiform region was also examined for a rigid lid. In this case a deep fast propagating circulation like the one previously discussed was superimposed on a slower propagating circulation characterized by a mid-level inflow and upper- and lower-level outflows. This second slower moving mode had a cool potential temperature anomaly at low levels and a warm potential temperature anomaly aloft. The leading pulses of vertical motion had upward motion at low levels and downward motion aloft. The speed of the modes is given by

$c=\frac{N H}{n \pi}$,

where $N$ is the Brunt-Väisälä frequency, $H$ the height of the rigid lid and $n$ the vertical mode of the heating profile. This profile has the form $\sin (n \pi z / H)$, where $z$ is height.

The two-dimensional solution for a semi-infinite region, without a troposphere-stratosphere interface, shows consid- erable differences of the low-level fields in some respects (Pandya et al., 1993). In particular, the magnitude of the subsidence is substantially reduced, and it occurs over a much broader region. Moreover, the axis of the peak vertical velocity in the low-level subsidence region is no longer vertically aligned, but strongly tilted. Nevertheless, adiabatic warming behind the broader wave front still gradually approaches the values at the heated center. Another factor to consider is that in the real atmosphere there is increased stability above the tropopause, which partially reflects waves and to some extent increases the similarity to the rigid lid solution. An early twodimensional numerical simulation of a squall line showed a structure qualitatively similar to the first mode during the early stage of development (Nicholls, 1987). The deep convective heating extending to the top of the troposphere produced a deep overturning circulation with surface mesolows growing laterally away from the center of the convection at a rapid pace. For the first deep convective mode that extends throughout the depth of the tropical troposphere, $H$ is approximately $15 \mathrm{~km}$ and taking $N=0.01 \mathrm{~s}^{-1}$ gives a horizontal propagation speed of $48 \mathrm{~m} \mathrm{~s}^{-1}$. For the second mode the speed is $24 \mathrm{~m} \mathrm{~s}^{-1}$. So, the first mode is very fast moving and while the second mode is considerably slower its speed is still quite fast compared to typical atmospheric motions.

Mapes (1993) postulated that higher-order modes of the heating profile in an MCS may cause upward displacements at low levels in the nearby atmosphere, thus favoring the development of additional convection nearby. He also emphasized that the wave-like disturbances are not ordinary gravity waves, and, pointing out their similarity to tidal bores in water, referred to them as buoyancy bores. There has been a considerable amount of research since these earlier studies that has examined their role in convective systems (e.g., Pandya and Durran, 1996; McAnelly at al., 1997; Pandya et al., 2000; Nicholls and Pielke, 2000; Shige and Satomura, 2001; Lane and Reeder, 2001; Haertel and Johnson, 2001; Fovell, 2002; Liu and Moncrieff, 2004; Fovell et al., 2006; Tulich and Mapes, 2008; Bryan and Parker, 2010; Lane and Zhang, 2011; Adams-Selin and Johnson, 2013). Inclusion of planetary rotation confines the compensating subsidence and adiabatic warming caused by deep convection to a finite distance, measured by the Rossby radius of deformation (Bretherton, 1987, 1988; Liu and Moncrieff, 2004). Adjustment towards geostrophic balance when a spectrum of inertial-gravity wave modes is generated by a heat source has been investigated in a two-dimensional framework by Liu and Moncrieff (2004).

To the author's knowledge, the generation of thermally forced gravity waves, by radiative forcing, rather than latent heating, has not been examined before. The modulations of radiative forcing are generally quite slow, and the circulations generated very weak, so it would be unlikely that these propagating circulations could be directly observed, especially considering that they are typically superimposed on much stronger circulations. Nevertheless, they are evident 
in the idealized numerical experiments that are discussed in this study and it is useful to consider their properties in order to obtain a more complete understanding of the circulations generated by radiative forcing.

Recently Dunkerton et al. (2009) developed the "marsupial paradigm" that provides a theoretical framework for understanding tropical cyclogenesis from easterly waves. The Kelvin cat's eye within the critical layer, or "wave pouch", was identified as a favorable environment for tropical cyclogenesis. The theory is supported by both observations of a developing Pacific easterly wave (Montgomery et al., 2010; Raymond and Lopez Carrillo, 2011) and cloud-resolving numerical simulations (Zhang et al., 2011; Montgomery et al., 2010, 2012; Wang et al., 2010, 2012). A numerical model investigation of the thermodynamic aspects near the center of the wave pouch found that it was characterized by a high saturation fraction (Wang, 2012). It was hypothesized that updrafts were more vigorous in this region because of reduced dry air entrainment, and that this was favorable for tropical cyclogenesis. This present study makes a similar argument for the effect of enhanced relative humidity in the tropical disturbance due to radiative forcing. Support for the hypothesis is provided by recent cloud model experiments that indicate that for non-supercell environments a major effect of dry air aloft is to reduce the intensity of convection, including total condensation and rainfall (James and Markowski, 2009; Kilroy and Smith, 2013).

The approach that will be used in this study will be to start off by examining the response of an initially motionless atmosphere to a prescribed cooling rate, with a magnitude typical of radiative forcing, applied in the large-scale environment surrounding a non-forced core, which represents the region occupied by a cloud cluster. For these simulations radiation, microphysics and surface flux schemes are de-activated. Now it is obviously a considerable idealization to set radiative forcing to zero in the region representing the cloud cluster. We will show later in a full physics simulation of a system that evolves into a tropical cyclone, that the radiation scheme produces in the cloud cluster reduced longwave cooling at low levels, slight warming at mid levels, and very large magnitude forcing at cloud top and in the outer region of the sloping stratiform cloud base. Nevertheless, this simplified framework does give insight into the circulations expected to be induced by horizontal differential radiative forcing at low and mid levels between a cloud cluster and its environment. It is also worth noting that Gray and Jacobson (1977) presented estimated day and night radiative forcing rates in the clear sky and in the disturbance, which for the disturbance were very small beneath $400 \mathrm{mb}$ (Fig. 13 of their manuscript). A set of sensitivity experiments using this idealized approach is conducted, and then numerical experiments become progressively more complex, culminating in an examination of the influence of radiation in cloud-resolving full physics simulations of tropical cyclognesis. These latter full physics experiments do not explicitly show the mechanisms causing radi- ation to influence tropical cyclogenesis, but drawing on the results of the idealized simulations, some strong inferences can be made. The combined results of these numerical model experiments suggest that increased relative humidity caused by large-scale environmental radiative cooling in situ does play a significant role in accelerating the rate of tropical cyclogenesis, as other studies have similarly demonstrated its importance for increasing the intensity of MCSs. However, there is probably a more important role played by the circulations induced by horizontal differential radiative forcing, both in accelerating the rate of genesis and in causing diurnal cycles of convective activity. The larger magnitude forcing aloft appears to have less important impacts on these two aspects, but this study does not focus on upper-level forcing, so its potential importance for tropical cyclones is left for future research.

An outline of the remaining paper is as follows: in Sect. 2 the numerical model is described. In Sect. 3 the designs of the numerical experiments are discussed as well as the particular motivations for them. Results are presented in Sect. 4 and conclusions in Sect. 5.

\section{Numerical model}

RAMS is a nonhydrostatic numerical modeling system comprising time-dependent equations for velocity, nondimensional pressure perturbation, ice-liquid water potential temperature (Tripoli and Cotton, 1981), total water mixing ratio and cloud microphysics. The microphysics scheme has categories for cloud droplets, rain, pristine ice crystals, snow, aggregates, graupel and hail (Walko et al., 1995). There have been several improvements to the model physics incorporated since the NM13 study. A two-moment microphysical scheme is now used for all hydrometeor species (Meyers et al., 1997). Additionally, an improved scheme for cloud-droplet riming that uses a binned approach is employed (Saleeby and Cotton, 2008). Another change is that the surface parameterization of heat, vapor and momentum fluxes utilizes recent results of the Coupled Boundary Layer Air-Sea Transfer Experiment (CBLAST) presented by Bell (2012).

The radiation scheme used in this study is two-stream, and treats the interaction of three solar and five infrared bands with the model gases and cloud hydrometeors (Harrington, 1997; Harrington et al., 1999). This parameterization solves the radiative transfer equations for the three gaseous constituents, $\mathrm{H}_{2} \mathrm{O}, \mathrm{O}_{3}$ and $\mathrm{CO}_{2}$, and for the optical effects of the hydrometeor size spectra. The fast exponential sum-fitting of transmissions method of Ritter and Gelyn (1992) is used for gaseous absorption. The optical properties of water drops are calculated using Lorenz-Mie theory and for non-spherical ice crystals the theory of Mitchell et al. (1996) is used. Since the parameterization includes interaction with liquid and ice 
Table 2. Experiments with prescribed thermal forcing.

\begin{tabular}{|c|c|}
\hline Experiment & Description \\
\hline 1 & $\begin{array}{l}\text { Maximum cooling aloft: environmental cooling for } r>200 \mathrm{~km}, z<16 \mathrm{~km} \text {, with maximum am- } \\
\text { plitude at } 8 \mathrm{~km} \text {. }\end{array}$ \\
\hline 2 & $\begin{array}{l}\text { Maximum cooling aloft and at low levels: environmental cooling for } r>200 \mathrm{~km}, z<16 \mathrm{~km} \text {, } \\
\text { with maximum } \\
\text { amplitude at } 8 \mathrm{~km} \text {, and a secondary maximum at the surface. }\end{array}$ \\
\hline 3 & Uniform cooling: uniform environmental cooling for $r>200 \mathrm{~km}$, and $z<10 \mathrm{~km}$. \\
\hline 4 & Small annulus: uniform cooling between $r=200$ and $400 \mathrm{~km}$, and $z<10 \mathrm{~km}$. \\
\hline 5 & Large annulus: uniform cooling between $r=200$ and $1000 \mathrm{~km}$, and $z<10 \mathrm{~km}$. \\
\hline 6 & Wide unforced region: uniform environmental cooling for $r>600 \mathrm{~km}$, and $z<10 \mathrm{~km}$. \\
\hline 7 & $\begin{array}{l}\text { Diurnal forcing: idealized diurnal oscillation of uniform environmental forcing for } r>200 \mathrm{~km} \text {, } \\
\text { and } z<10 \mathrm{~km} \text {. }\end{array}$ \\
\hline 8 & $\begin{array}{l}\text { Core forcing: uniform warming between } z=5 \text { and } 10 \mathrm{~km} \text {, uniform cooling below } 5 \mathrm{~km} \text {, for } \\
r<200 \mathrm{~km} \text {. }\end{array}$ \\
\hline 9 & $\begin{array}{l}\text { Weak vortex: uniform environmental cooling for } r>200 \mathrm{~km}, z<10 \mathrm{~km} \text {, and a vortex with } \\
\text { surface winds of } 12 \mathrm{~m} \mathrm{~s}^{-1} \text {. }\end{array}$ \\
\hline 10 & $\begin{array}{l}\text { Strong vortex: uniform environmental cooling for } r>200 \mathrm{~km}, z<10 \mathrm{~km} \text {, and a vortex with } \\
\text { surface winds of } 30 \mathrm{~m} \mathrm{~s}^{-1} \text {. }\end{array}$ \\
\hline
\end{tabular}

hydrometeor size spectra, this enables radiation to respond to variations in droplet size spectra.

A standard first-order subgrid-scale turbulence scheme developed by Smagorinsky (1963) is used with modifications by Lilly (1962) and Hill (1974) that enhance diffusion in unstable conditions and reduces diffusion in stable conditions. RAMS utilizes the two-way interactive multiple nested grid scheme developed by Clark and Farley (1984). The radiation boundary condition described by Klemp and Wilhelmson (1978) is used at the lateral boundary of the coarse grid. A Rayleigh friction layer is included at upper levels.

\section{Description of experiments}

The experiments conducted in this study can be classified into three categories. First are idealized experiments without radiation, cloud microphysics or surface fluxes. A list of the ten main experiments in this category is given in Table 2. Cooling or heating rates that represent idealized radiative forcing are prescribed. For many of the experiments a constant cooling is prescribed at the initial time in an environment that surrounds a core region, approximately the size of a cloud cluster, which is absent of any forcing. It is shown that thermally generated gravity waves, or buoyancy bores, propagate quickly into the core and induce upward motion, resulting in significant changes in the core temperature and relative humidity. Experiments are conducted for various vertical profiles of the cooling. Additional sensitivity tests examine the response for cooling in annular regions of two different breadths surrounding the core. This is to see how large the cloud-free environment needs to be in order to generate significant sustained upward motion in the core. The sensitivity to the width of the core is also examined by increas- ing it from 200 to $600 \mathrm{~km}$. This has relevance for contrasting how the response may be different for a small tropical cyclone versus a large one. Then a simple idealized representation of diurnal environmental forcing is used to examine how the core responds to changes in the environmental forcing. This is followed by an experiment to examine the response to forcing within the core, which has a warming region above a cooling region. The reason for this experiment, as previously remarked upon, is that the full physics simulation with radiation included shows a warming at mid levels and a cooling at low levels in the core. Also included at this stage are simulations with two vortices of different strengths to examine how the presence of a vortex influences the induced upward motion in the core. It was found that using a sharp transition between the forced and unforced regions leads to some noise in the fields. To avoid this a linear ramp $50 \mathrm{~km}$ wide is used. For instance, for a typical setup that might be designated "unforced for radius less than $200 \mathrm{~km}$ ", the region $175 \mathrm{~km}$ is actually unforced and linearly changes to the fully forced value at a radius of $225 \mathrm{~km}$. The designation of "unforced for radius less than $200 \mathrm{~km}$ " is used for brevity.

The second category of experiments has the radiation scheme turned on, but microphysics and surface fluxes turned off. Sensitivity to whether or not radiation is included in the core is examined when there is an initial mid-level vortex present identical to the one used to initialize the full physics experiments. The two experiments in this category are listed in Table 3. The first experiment, which has radiation activated in the core region as well as in the environment, is relevant for the early development of the full physics simulations because it takes some time before deep convection develops and for the cloud canopy to form. The second experiment, which has no radiation in the core, is an idealization, 
Table 3. Radiative transfer scheme activated with a mid-level vortex and a moistened core.

\begin{tabular}{ll}
\hline Experiment & Description \\
\hline 11 & $\begin{array}{l}\text { Radiative transfer scheme activated in the whole domain, with a weak mid-level vortex and } \\
\text { a moistened core. }\end{array}$ \\
& $\begin{array}{l}\text { Radiative scheme activated in the environment for } r>200 \mathrm{~km} \text {, with a weak mid-level vortex } \\
\text { and a moistened core. }\end{array}$ \\
\hline
\end{tabular}

Table 4. Experiments with full physics.

\begin{tabular}{ll}
\hline Experiment & Description \\
\hline 13 & Radiation in the whole domain. \\
14 & No radiation. \\
15 & Radiation only in the environment, $r>200 \mathrm{~km}$. \\
16 & Radiation only in the core, $r<200 \mathrm{~km}$. \\
17 & Prescribed uniform environmental cooling, for $r>200 \mathrm{~km}, z<10 \mathrm{~km}$. \\
18 & Radiation only in a large core, $r<400 \mathrm{~km}$. \\
19 & Radiation only in the environment outside a large unforced region, $r>500 \mathrm{~km}$. \\
20 & No radiative forcing below $1.5 \mathrm{~km}$. \\
21 & No radiative forcing aloft above $8 \mathrm{~km}$. \\
22 & Prescribed horizontally homogeneous cooling throughout the domain, uniform below $10 \mathrm{~km}$. \\
23 & Radiative cooling only. \\
24 & Radiative warming only. \\
\hline
\end{tabular}

but gives an idea of what circulations and resultant effects caused by radiative forcing outside the core region might be expected to occur in the lower and middle levels of the troposphere when a cloud canopy forms. The simulation begins in the mid-morning and longwave cooling is negated to a large degree by shortwave heating throughout the day. During the nighttime it will be shown that a substantial increase in core humidity occurs for Experiment 12.

The third category is the full physics simulations that include both microphysics and surface fluxes. The main focus of these experiments is to provide further supporting evidence that the differential radiative forcing mechanism is playing a primary role, once a thick cloud canopy forms that is opaque in the infrared. A list of the twelve experiments is given in Table 4. The first five experiments are treated as a group. The first simulation, Experiment 13, with radiation included everywhere in the domain is examined in some detail. It is shown how a small tropical cyclone develops quickly along pathway Two (NM13). The following sensitivity tests are carried out: (1) radiation is turned off everywhere. This experiment demonstrates the difference in the rate of genesis caused by radiative forcing; (2) radiation is turned off in the core (radius less than $200 \mathrm{~km}$ ). This experiment is conducted to show that it is likely that mean environmental radiative cooling is having a significant effect on the rate of genesis. Prior to the canopy shield extending beyond a radius of $200 \mathrm{~km}$ the radiative forcing can be expected to be similar to Experiment 12, and it can be seen whether the induced upward motion in the core and enhanced humidity in- fluences the intensity of convection; (3) radiation is turned off in the environment but included in the core. Again, this is to reinforce the conclusion that environmental radiative cooling is important, since the result for this case will be seen to be a slow genesis rate. Together Experiments 15 and 16 will make a strong case for differential forcing being important and the direct cloud-radiative interactions aloft as being relatively unimportant in influencing the rate of development of the system; (4) radiation is turned off and a constant uniform cooling is applied outside the core, below a height of $10 \mathrm{~km}$. This is the same cooling function as used in Experiment 3. The objective is to demonstrate that the changes in the core due to this constant applied differential thermal forcing have a major impact on the genesis rate. The development of the low-level wind speed is compared for the five cases to see which develop into tropical cyclones the quickest. Also, the time evolution of the total mass of hydrometeors is compared to see which cases develop significant oscillations of convective activity. The following seven experiments shown in Table 4 were conducted in order to clarify some issues brought up by results of the previous experiments. The reasoning behind these experiments and relation to some of the idealized experiments will be explained in the results section.

For the majority of the first category of experiments, only one grid is used. The horizontal grid increment is $12 \mathrm{~km}$, with $(x, y, z)$ dimensions of $170 \times 170 \times 48$. The vertical grid increment is $60 \mathrm{~m}$ and gradually stretched with height to the top of the domain at $z=22.3 \mathrm{~km}$. The depth of the upper Rayleigh friction layer is $6 \mathrm{~km}$. The horizontal dimensions of 
the grid were increased in size for the large annulus experiment to 400 grid points. For the vortex simulations a better horizontal resolution was necessary to resolve adequately the vortex and a nested grid was added with a horizontal grid increment of $3 \mathrm{~km}$ and $(x, y, z)$ dimensions of $202 \times 202 \times 48$.

The second category of experiments also includes a nested grid with the same horizontal grid increments and horizontal dimensions as for the previous vortex experiments. The number of vertical grid points is increased to 56 to be consistent with the full physics simulations. In this case the vertical grid increment is gradually stretched from $60 \mathrm{~m}$ but not allowed to exceed $700 \mathrm{~m}$, which occurs at approximately a height of $9 \mathrm{~km}$, and is thereafter held constant to the top of the domain at $z=22.9 \mathrm{~km}$. The better resolution aloft was deemed necessary for the full physics simulations, because the canopy top is near the tropopause and plays a radiatively active role.

For the full physics simulations of the third category, three grids are used with horizontal grid increments of 24 , 6 , and $2 \mathrm{~km}$, and $(x, y, z)$ dimensions of $150 \times 150 \times 56$, $150 \times 150 \times 56$, and $203 \times 203 \times 56$, respectively. Each grid is centered within the next coarsest grid.

The temperature and moisture profiles used to initialize the model are the mean Atlantic hurricane season sounding of Jordan (1958) which is slightly moister at low levels than used by NM13. The details of the procedure used for the initial vortex are discussed by Montgomery et al. (2006). For the full physics simulations the initial mid-level vortex is similar to that shown in Fig. 1 of NM13, with maximum winds of $8 \mathrm{~m} \mathrm{~s}^{-1}$ at a radius $r=75 \mathrm{~km}$ and a height of $z=4 \mathrm{~km}$. The core of the vortex is moistened to $85 \%$ of saturation with respect to water below $8 \mathrm{~km}$. The model is configured for an f-plane at latitude of $15^{\circ}$. The center of the domain for these simulations is at a longitude of $-40^{\circ}$. The shortwave radiation computation accounts for the longitudinal variation of the solar zenith angle. All simulations are begun at 1200 GMT. The sea surface temperature for the full physics simulations is set to a constant value of $29^{\circ} \mathrm{C}$.

\section{Results}

\subsection{Idealized experiments with prescribed forcing}

In the first experiment a cooling rate is specified for a radius greater than $200 \mathrm{~km}$ from the center of the domain, and has maximum amplitude at a height of $8 \mathrm{~km}$. The cooling extends from the surface where the cooling rate is $-1.2 \times 10^{-5} \mathrm{~K} \mathrm{~s}^{-1}$ up to a height of $16 \mathrm{~km}$. The maximum amplitude of the cooling at $8 \mathrm{~km}$ is $-2.8 \times 10^{-5} \mathrm{~K} \mathrm{~s}^{-1}$. Figure 1 shows $x / z$ vertical sections through the center of the domain of potential temperature perturbation, pressure perturbation, $x$ component of velocity $\mathrm{u}$, and vertical velocity at $50 \mathrm{~min}$. The cooling has begun to produce a decrease in the temperature of the environment. A laterally propagating wave-like circulation has formed at the boundary between the cooled and non-
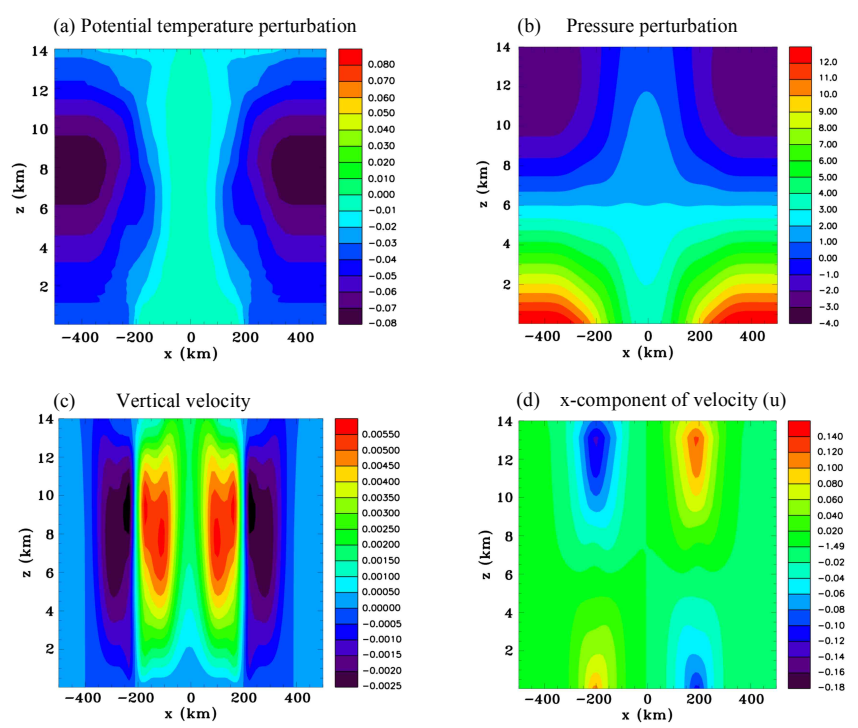

Figure 1. Vertical sections for Experiment 1: the maximum cooling aloft case, at $t=50 \mathrm{~min}$. (a) Potential temperature perturbation (K), (b) pressure perturbation $(\mathrm{mb}),(\mathbf{c})$ vertical velocity $\left(\mathrm{m} \mathrm{s}^{-1}\right)$, and (d) $x$-component of velocity, $u\left(\mathrm{~m} \mathrm{~s}^{-1}\right)$.

cooled regions. A region of upward motion is propagating towards the center, whereas a region of downward motion is propagating into the environment. The circulation has a similarity to the two-dimensional solutions obtained by Nicholls et al. (1991), but clearly the three-dimensional geometry of this simulation has some important implications. For instance, the ring of upward motion near the center is stronger in magnitude than the ring of downward motion in the environment. The circulation is associated with a high-pressure perturbation at the surface and a low-pressure perturbation aloft that is propagating towards the center. The uplift is causing the air to cool adiabatically, creating a bulge of cold air in the core at mid-tropospheric levels where the upward motion is strongest. The upward motion has a maximum of approximately $6 \mathrm{~mm} \mathrm{~s}^{-1}$ and the horizontal motions, while considerably larger with a magnitude of approximately $0.1 \mathrm{~m} \mathrm{~s}^{-1}$, are also not particularly strong. The ring of downward motion that propagates outward into the environment causes adiabatic warming that offsets to some extent the applied cooling. However, the wave amplitude decreases rapidly with distance travelled and, because the wave is propagating, its impact at any location is transient, whereas the environmental cooling is persistent. Figure 2 shows fields at $12 \mathrm{~h}$. A sustained weak upward motion in the unforced region has led to cooling at the center, which above $3 \mathrm{~km}$ is very similar to that of the environment. A maximum perturbation of over $1 \mathrm{~K}$ occurs just below a height of $8 \mathrm{~km}$. The relative humidity has increased significantly as well, particularly near the surface and further aloft within the region of maximum upward motion, with an increase of over $6 \%$. Therefore, in spite of the vertical motion being relatively weak over the course of a $12 \mathrm{~h}$ period, 

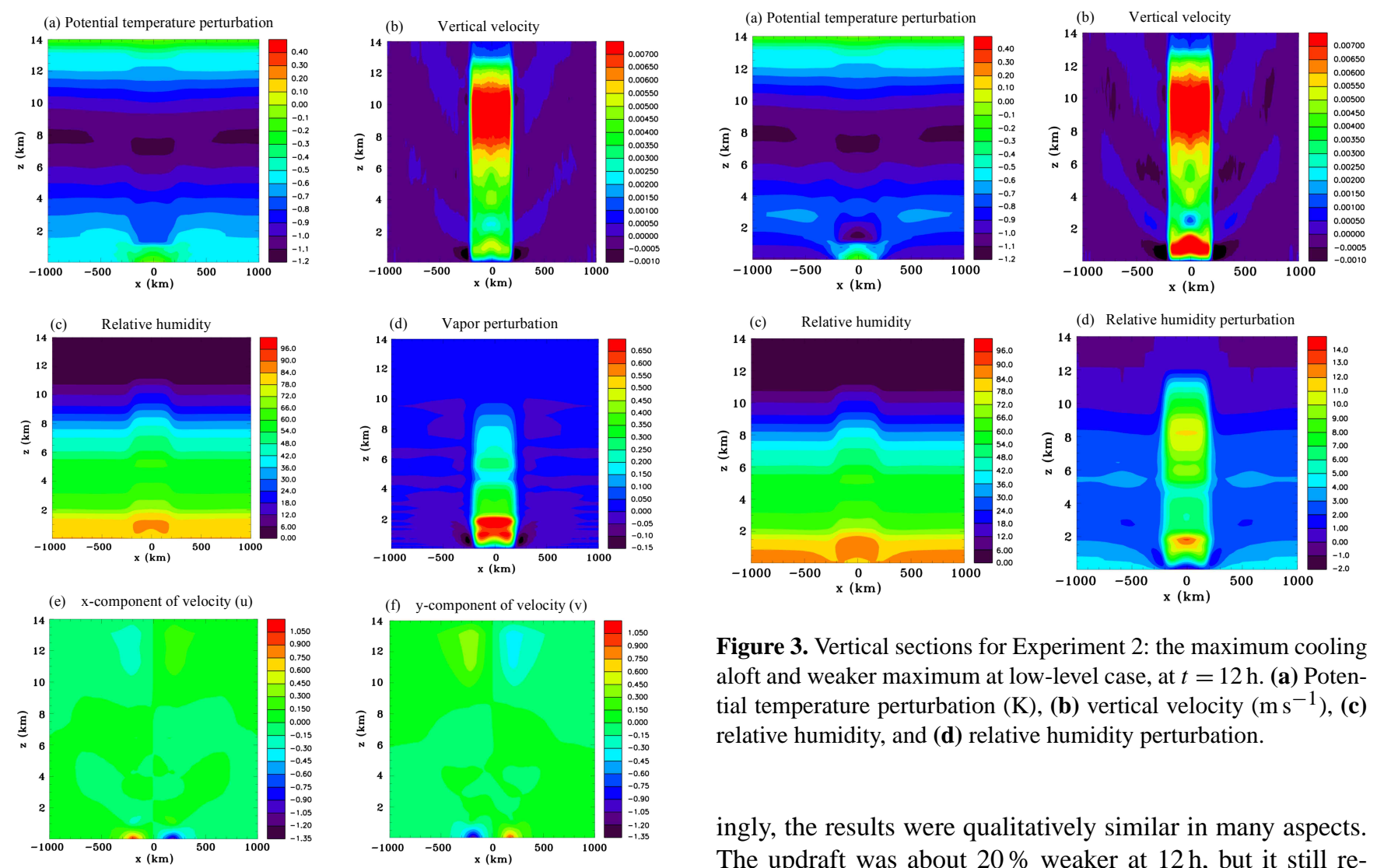

Figure 2. Vertical sections for Experiment 1: the maximum cooling aloft case, at $t=12 \mathrm{~h}$. (a) Potential temperature perturbation (K), (b) vertical velocity $\left(\mathrm{m} \mathrm{s}^{-1}\right)$, (c) relative humidity, (d) vapor perturbation $\left(\mathrm{g} \mathrm{kg}^{-1}\right)$, (e) $x$-component of velocity, $u\left(\mathrm{~m} \mathrm{~s}^{-1}\right)$, and (f) $y$-component of velocity, $v\left(\mathrm{~m} \mathrm{~s}^{-1}\right)$.

significant changes result in the core. Figure 2d, showing the vapor mixing ratio anomaly, indicates that the vertical motion has produced the largest change in the lowest $2 \mathrm{~km}$ of approximately $0.7 \mathrm{~g} \mathrm{~kg}^{-1}$ where the vertical gradient of moisture in the Jordan sounding is largest (not shown). There is a small increase in the low-level relative humidity near the surface in the environment, which must be due to the cooling since there is no increase in the environmental vapor mixing ratio. Figure $2 \mathrm{e}$ shows that by far the strongest horizontal motions occur near the surface at a radius of $200 \mathrm{~km}$. The surface inflow at $12 \mathrm{~h}$ is fairly substantial, with a magnitude of approximately $0.8 \mathrm{~m} \mathrm{~s}^{-1}$, which is considerably stronger than the horizontal motions that occurred at $50 \mathrm{~min}$ associated with the inward propagating wave-like feature shown in Fig. 1. Due to the Coriolis force there has been a small but significant increase in the cyclonic winds at the same location of approximately $1 \mathrm{~m} \mathrm{~s}^{-1}$ (Fig. 2f).

This simulation was also run in two dimensions to see whether there was a significant three-dimensional aspect to the relatively strong updraft in the unforced core. Interest-

Figure 3. Vertical sections for Experiment 2: the maximum cooling aloft and weaker maximum at low-level case, at $t=12 \mathrm{~h}$. (a) Potential temperature perturbation $(\mathrm{K}),(\mathbf{b})$ vertical velocity $\left(\mathrm{m} \mathrm{s}^{-1}\right),(\mathbf{c})$ relative humidity, and (d) relative humidity perturbation.

ingly, the results were qualitatively similar in many aspects. The updraft was about $20 \%$ weaker at $12 \mathrm{~h}$, but it still resulted in significant changes to relative humidity and potential temperature in the core. A major difference was that the deep pulses of subsidence propagating towards the boundaries were much more evident since they did not decrease in amplitude so quickly. The leading edges of the pulses reached the open boundaries at approximately $4.5 \mathrm{~h}$ and then propagated through them. The distance the pulses travelled to the lateral boundaries from where they formed at $200 \mathrm{~km}$ from the center was about $800 \mathrm{~km}$. This gives a propagation speed of about $49 \mathrm{~m} \mathrm{~s}^{-1}$. The pulses were about $14 \mathrm{~km}$ deep, so the speed seems in reasonable agreement with the speed of the first mode estimated from Eq. (1), discussed in the introduction, to be about $48 \mathrm{~m} \mathrm{~s}^{-1}$ for a $15 \mathrm{~km}$ deep wave.

Figure 3 shows results for the second experiment at $12 \mathrm{~h}$ that has the same environmental cooling as the first except there is enhanced cooling near the surface. This case produces increased low-level upward motion in the core that increases the low-level relative humidity, with the largest perturbation occurring just below $2 \mathrm{~km}$ (Fig. $3 \mathrm{~d}$ ). Also, the increased environmental cooling near the surface produces a notable increase in the low-level relative humidity in the environment.

These two preliminary experiments suggest that in order to understand the effects of radiation on a cloud cluster that has a canopy capable of significantly reducing longwave cooling, account needs to be taken of the thermally driven circulations caused by differential heating or cooling between the cloud cluster and the environment. These idealized simulations re- 
sulted in significant changes to the relative humidity, lapse rates and surface cyclonic circulation in the unforced core of the system. In addition to the changes caused by the induced circulations there was a notable increase in low-level relative humidity in the environment caused by the environmental cooling in Experiment 2, which could also influence the development of convection. The latter is the second mechanism of radiative influence on cloud clusters discussed in the introduction.

Experiment 3 examines the response to an environmental cooling that is uniform beneath $10 \mathrm{~km}$ with a value of $-1.5 \mathrm{~K}$ day $^{-1}$. In Fig. 4 this case is compared at $12 \mathrm{~h}$ with Experiment 4 , which has the same uniform cooling rate prescribed beneath $10 \mathrm{~km}$ in an annulus between 200 and $400 \mathrm{~km}$ from the center. Although the air in the annulus became colder during the first hour, this did not persist, and by $12 \mathrm{~h}$ it can be seen that there are only weak temperature changes in the annulus. There is a weak cooling at the surface, but much of the air in the annulus is actually very slightly warmer. The case with cooling throughout the environment is similar to the first two experiments, but is more uniform in the vertical, except at low levels in the unforced core. There is weak downward motion of a few $\mathrm{mm} \mathrm{s}^{-1}$ in the cooled annulus and this results in a significant decrease in relative humidity. This is due to downward advection of drier air, which is evident since the small potential temperature change in the annulus is not enough to significantly influence the humidity except near the surface. The decrease in potential temperature next to the surface is due to the applied cooling, which is not offset much by adiabatic warming since the subsidence there is small. This results in an increase in humidity in a very thin layer adjacent to the surface. In the core the upward motions are considerably less at $12 \mathrm{~h}$ for the annulus case and there are regions of downward motion at $z=2$ and $7 \mathrm{~km}$. There is an increase in surface cyclonic flow, but it is not as large as for the case with cooling throughout the environment. There is also anticyclonic flow at the surface at a radius of $400 \mathrm{~km}$.

Experiment 5 has a much larger annulus that extends to a radius of $1000 \mathrm{~km}$. Figure 5 shows results at $5 \mathrm{~h}$. At this stage the air is colder in the cooled annulus, but a very deep region of subsidence is evident travelling from the edge of the outer annulus boundary inwards towards the center that is tending to warm adiabatically the air in the annulus, counteracting the diabatic cooling below $10 \mathrm{~km}$, as well as warming the air above $10 \mathrm{~km}$. At the same time a region of upward motion that is weaker in magnitude can be seen propagating into the environment. At the inner annulus boundary a ring of upward motion propagated into the core and caused a strong sustained upward motion as seen in Fig. 5b, similarly to the other cases. A ring of downward motion also propagated outwards from the inner annulus boundary and is superimposed with the inward propagating downward motion. It is smaller in magnitude, however, so the inward propagating downward motion is more evident. It is clear that this inward propagating downward motion is going to significantly influence the
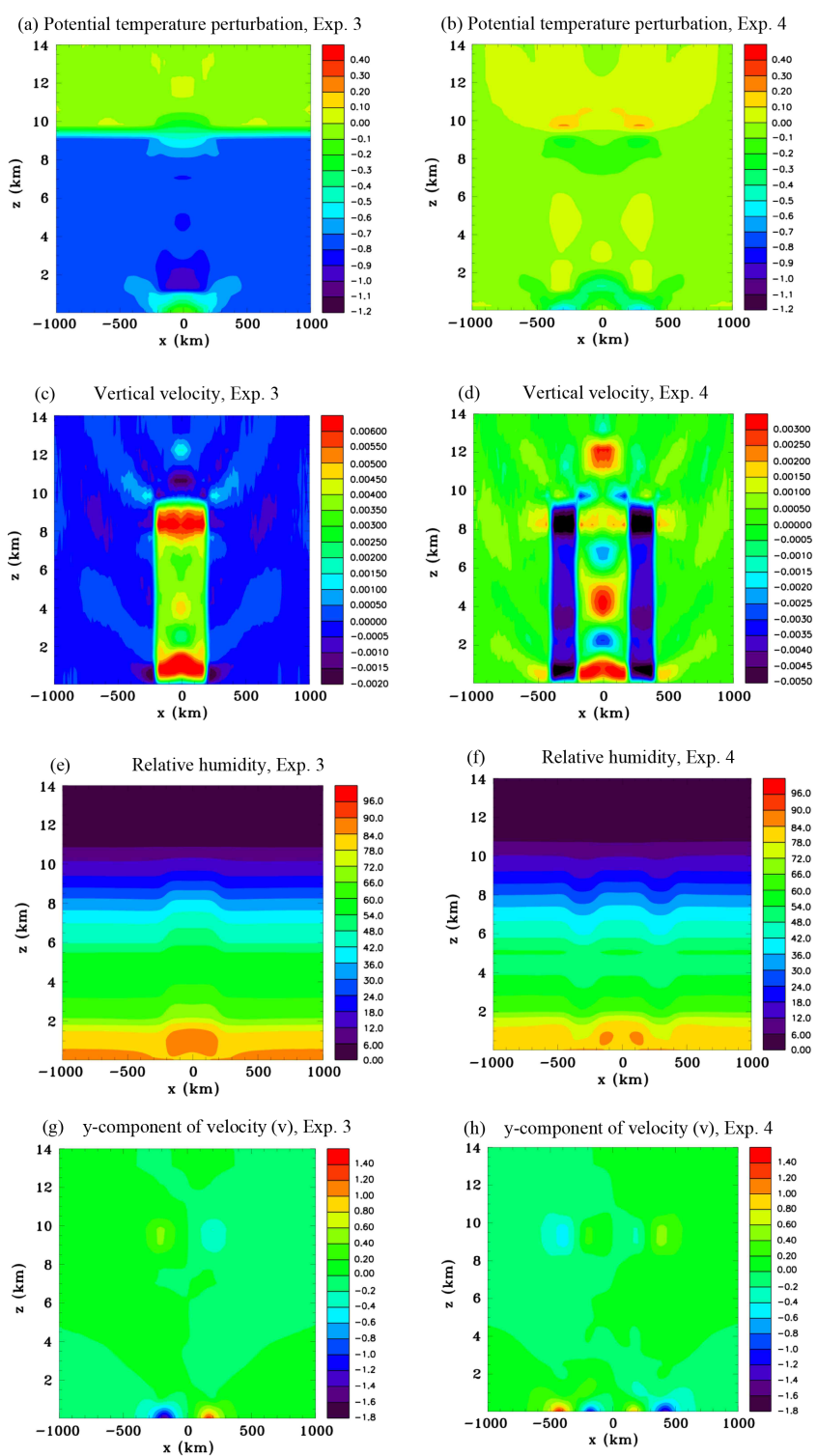

Figure 4. Vertical sections comparing Experiment 3: the uniform cooling case with Experiment 4, the small annulus case, at $t=12 \mathrm{~h}$. (a) and (b) potential temperature perturbation (K), (c) and (d) vertical velocity $\left(\mathrm{m} \mathrm{s}^{-1}\right),(\mathbf{e})$ and (f) relative humidity, and (g) and (h) $y$-component of velocity, $v\left(\mathrm{~m} \mathrm{~s}^{-1}\right)$, for Experiments 3 and 4, respectively.

vertical velocities in the center when reaching it. This situation also occurred for the $200 \mathrm{~km}$ annulus previously discussed, but is more easily seen for this larger annulus case. At this time there has been a significant increase in relative humidity in the center and a small decrease in the annulus.

Figure 6 shows results at $12 \mathrm{~h}$. There is colder air at low levels in the annulus and a cool region in the core at a height of about $1.5 \mathrm{~km}$. There is also a cold anomaly at the top of the annulus. The middle levels have not cooled significantly however either in the annulus or core. The core does not show 

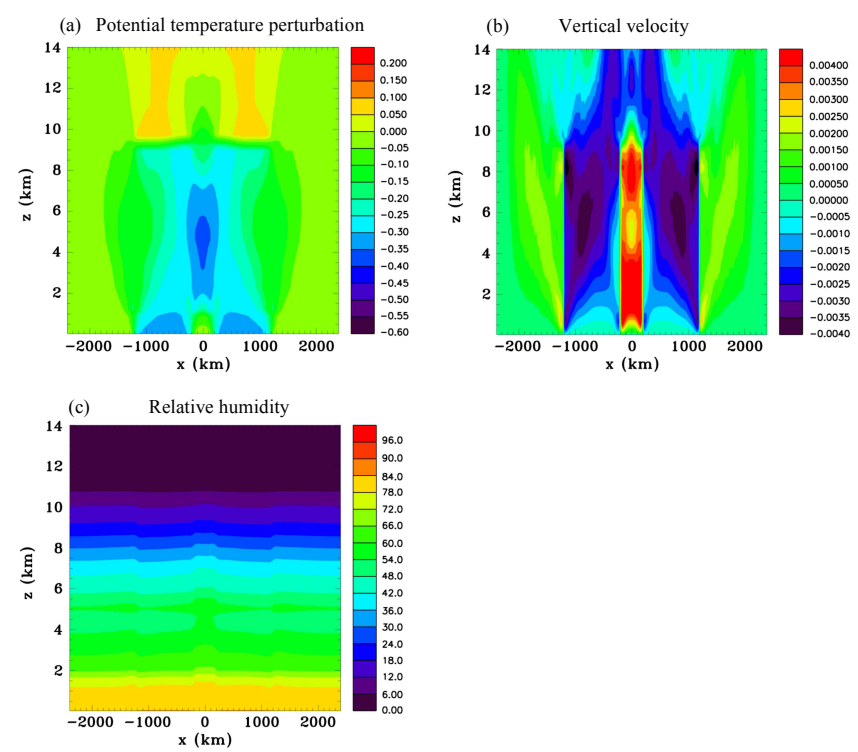

Figure 5. Vertical sections for Experiment 5: the large annulus case, at $t=5 \mathrm{~h}$. (a) Potential temperature perturbation (K), (b) vertical velocity $\left(\mathrm{m} \mathrm{s}^{-1}\right)$, and (c) relative humidity.

significant upward motion as it did at $5 \mathrm{~h}$ except close to the surface. There has been a significant increase in the relative humidity in the core, but not as large as for the case shown in Fig. 4e with the cooling throughout the environment. There is some increase in relative humidity at low levels in the annulus, where apparently the downward motion is too weak to counteract the increase due to cooling. The anticyclonic surface winds are considerably stronger at the outer boundary of the annulus than at the inner boundary.

The annulus experiments show that the response in the core becomes more significant as the size of the region that is cooled is increased as might be expected. They also show that the response can be quite complicated and that there can be significant drying within the cooled region due to subsidence. This case is extremely idealized and would not occur in nature, but is relevant to a full physics simulation of tropical cyclogenesis that will be discussed later. It further illustrates the fast propagation of these thermally generated circulations that travel in a wave-like manner and how the magnitude of the vertical velocity is increased or diminished depending on whether the direction of propagation is towards the center or away from it, respectively.

In light of the results of the annulus experiments two other simulations were run without an annulus, which will be briefly mentioned. It would appear that it is important to have a very large surrounding cloud-free environment for the region representing the cloud cluster to be significantly influenced by circulations induced by environmental cooling. A simulation was run similarly to Experiment 3, but with a much reduced domain size with a width of only $600 \mathrm{~km}$. Additionally, a second simulation was conducted for this re- duced grid size with the open boundary conditions replaced by cyclic boundary conditions. In spite of the reduced size of the domain, results for the first simulation were quite similar to Experiment 3, showing significant modification of the core due to induced upward motion. The open boundary conditions allowed the outward propagating pulse of downward motion to propagate without reflection when it reached the boundary and persistent low-level inflow and upper-level outflow at the boundary were established. The strong horizontal pressure gradients responsible for accelerating the inflow at low levels and outflow at high levels were for the most part still represented in the smaller domain simulation. So, it was not necessary to have a large domain size to simulate sustained upward motion in the core when radiative boundary conditions were used. One might say, however, that in the real atmosphere, if horizontal gradients in radiative forcing in the far environment surrounding a cloud cluster were to occur at some point in time, possibly due to clouds, they would generate circulations that would propagate towards the cluster, and depending on their strength and duration, could eventually have some influence on it. The effect of environmental cooling at night is therefore going to be more effective when there is a large region of clear sky surrounding the cloud cluster. The second simulation showed a significant difference with Experiment 3 since the pulse of outward propagating downward motion propagated back into the domain because of the cyclic boundary conditions. This resulted in less modification of the unforced core's relative humidity and potential temperature. This latter experiment could be considered to qualitatively mimic what might happen when there are nearby cloud clusters, which would apparently reduce the sustained upward motion in the core produced by nighttime clear-sky radiative cooling.

Experiment 6 examines the sensitivity to increasing the size of the unforced core region to a radius of $600 \mathrm{~km}$ for a uniform cooling below $10 \mathrm{~km}$ in the environment. Figure 7 shows that for this case the colder air at $12 \mathrm{~h}$ is less horizontally homogeneous than for a radius of $200 \mathrm{~km}$ (Fig. 4a). A minimum occurs at middle levels in the core. There is still a fairly significant increase in relative humidity in the core, although this is noticeably a maximum near its periphery. This result suggests that the center of a large tropical cyclone will be less impacted by the differential radiative forcing mechanism than a small one.

Experiment 7 investigates the response to an idealized diurnal oscillation in the cooling function in the environment. Figure 8 shows time series of the environmental forcing and potential temperature changes it produces both in the environment and the core. A uniform cooling is applied below $10 \mathrm{~km}$ and for a radius greater than $200 \mathrm{~km}$, which is constant for $12 \mathrm{~h}$ and then decreases in magnitude to zero by $18 \mathrm{~h}$ and increases in magnitude back to the original cooling rate by $24 \mathrm{~h}$ using a half-sine wave. This approximately represents a cooling at night due to infrared cooling and then a reduced net cooling rate in the daytime due to shortwave heating, 

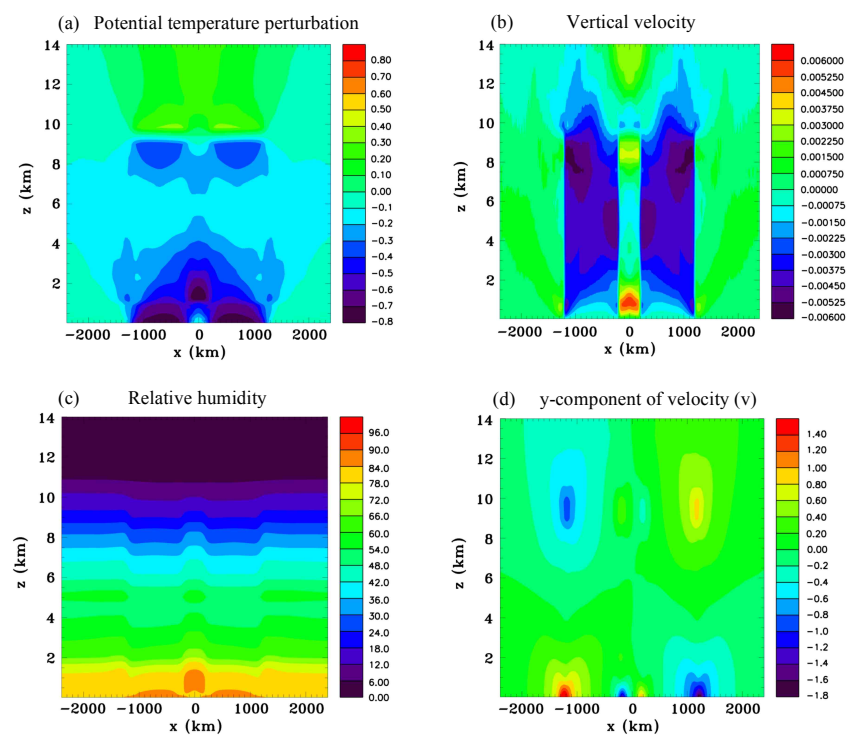

Figure 6. Vertical sections for Experiment 5: the large annulus case, at $t=12 \mathrm{~h}$. (a) Potential temperature perturbation (K), (b) vertical velocity $\left(\mathrm{m} \mathrm{s}^{-1}\right)$, (c) relative humidity, and (d) $y$-component of velocity, $v\left(\mathrm{~m} \mathrm{~s}^{-1}\right)$.

which is a maximum at noon. The simulation was run for $72 \mathrm{~h}$. Figure $8 \mathrm{~b}$ compares the potential temperature change at a height of $5 \mathrm{~km}$ in the environment with the change in the core at the same height. The first $12 \mathrm{~h}$ is identical to Experiment 3 (Fig. 4) that was compared to the annulus experiment. The temperature decreases first in the environment, but $2 \mathrm{~h}$ later it has decreased by as much in the center of the core. Later in the simulation the core temperature anomaly is actually slightly larger in magnitude than in the environment, although the reason for this behavior is unclear. The core temperature at this level is closely matching what happens in the environment with little time lag. During the nighttime there is a steady decrease in temperature and then a leveling off in the daytime.

Figure $9 \mathrm{a}-\mathrm{h}$ shows vertical sections at various times for the idealized diurnal cycle simulation. Figure 9a shows the vertical velocity at $18 \mathrm{~h}$, when the environmental cooling rate is zero, representing the middle of the day. The upward motion that existed at $12 \mathrm{~h}$ (Fig. 4c) has been replaced by very weak downward motion through most of the core below $10 \mathrm{~km}$. Figure $9 \mathrm{~b}$ shows the vertical velocity at $30 \mathrm{~h}$ in the middle of the night. Significant upward motion now extends below $10 \mathrm{~km}$ through the core except near the surface. Figure 9c shows the vertical velocity at $72 \mathrm{~h}$, which is at the end of the third day. There is downward motion near the surface in the core and a weak shallow outflow at the surface (Fig. 9g). The temperature of the air in the core is similar to that in the environment except at low levels (Fig. 9d). There has been a significant increase in the relative humidity both in the environment and in the core. In the environment it is due to the diabatic cooling, whereas in the core it is due to the forced
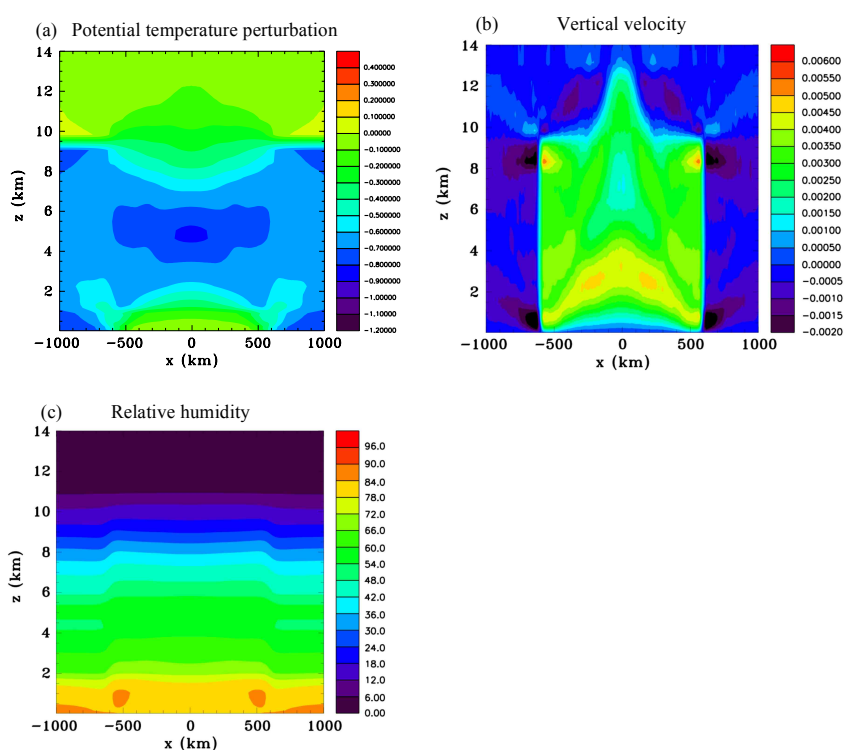

Figure 7. Vertical sections for Experiment 6: the wide unforced region case, at $t=12 \mathrm{~h}$. (a) Potential temperature perturbation (K), (b) vertical velocity $\left(\mathrm{m} \mathrm{s}^{-1}\right)$, and (c) relative humidity.

upward motion, which was strong in the three nighttime periods. The core relative humidity is larger than in the environment except at the surface. Similarly to Experiment 2 the perturbation of relative humidity from the initial state is largest just below $2 \mathrm{~km}$. Although the flow at $72 \mathrm{~h}$ is slightly divergent at the surface, there was mean low-level convergence during the simulation, which resulted in a notable increase in the cyclonic flow of approximately $3 \mathrm{~m} \mathrm{~s}^{-1}$ (Fig. 9f).

Results of this diurnal experiment suggest that if a cloud canopy forms aloft that reduces longwave cooling beneath it, then the environmental radiative forcing will induce a temperature tendency at mid levels in the core that will be similar to what occurs in the environment with only a small time lag. The temperature tendencies will follow the day/night modulations of the environment and except near the surface would also have the same vertical profile. The small time lag appears to be a consequence of the fast propagation speed of the thermally forced gravity wave modes. The temperature tendencies would presumably be superimposed on those occurring in the cloud system caused by latent heating or cooling, although this has not been demonstrated here. The induced mean upward motion tendency would increase the humidity in the core, most likely favoring the development of convection.

Experiment 8 examines the response to an upper-level heating above a low-level cooling for radius less than $200 \mathrm{~km}$. This forced core experiment has relevance because the full physics simulations show that when an extensive canopy forms aloft there is weak mid- to upper-level radiative heating and weak low-level cooling. One of the full physics simulations examines what happens when radiative forcing is 
(a)

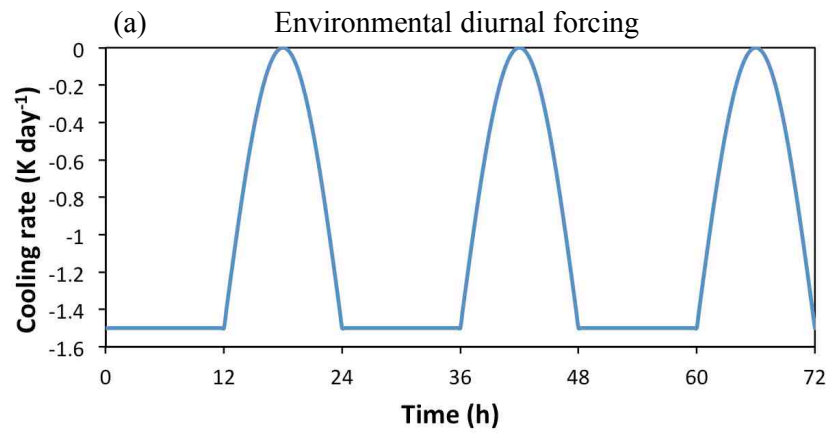

(b)

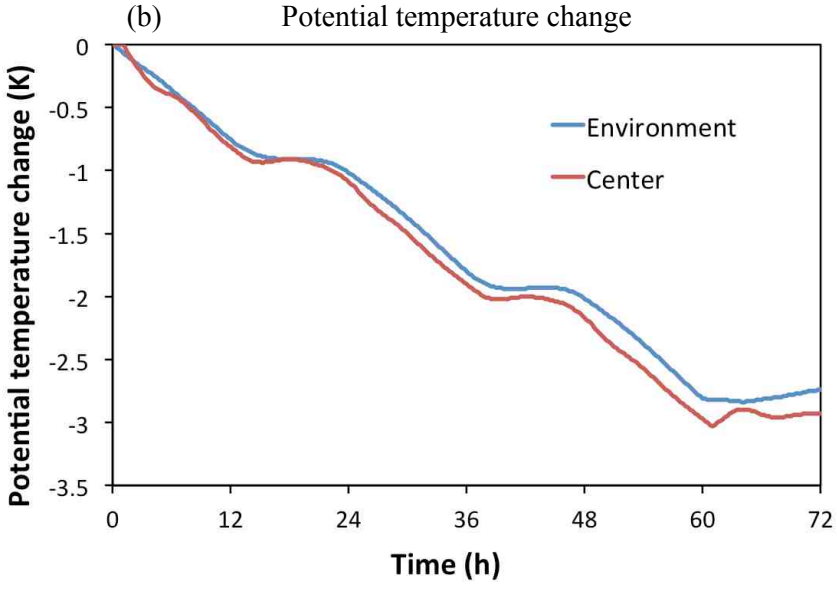

Figure 8. Time series for Experiment 7: the idealized diurnal forcing case. (a) Environmental forcing for $r>200 \mathrm{~km}, z<10 \mathrm{~km}$, and (b) potential temperature change in the environment at $r=660 \mathrm{~km}$ and $z=5 \mathrm{~km}$, and at the center of the unforced region at $z=5 \mathrm{~km}$.

only activated for a radius less than $200 \mathrm{~km}$ from the center, which results in a similar scenario. There has been no attempt to match the magnitude and spatial distribution of the heating aloft and cooling below with the full physics simulations. Instead, a uniform heating rate of $1.5 \mathrm{~K} \mathrm{day}^{-1}$ is specified between 5 and $10 \mathrm{~km}$ and a uniform cooling rate of $-1.5 \mathrm{~K} \mathrm{day}^{-1}$ between the surface and $5 \mathrm{~km}$, for a more straightforward comparison with some of the previous simulations. Figure 10a-f compares fields at 1 and $12 \mathrm{~h}$. Figure 10a shows that at $1 \mathrm{~h}$ there is air that is slightly warmer above air that is slightly colder, as might be expected from the spatial distribution of the heating/cooling function. The magnitude of heating/cooling matches what is expected from the applied forcing $\sim 0.06 \mathrm{~K} \mathrm{~h}^{-1}$. Figure $10 \mathrm{~b}$ shows, however, that at $12 \mathrm{~h}$ this pattern has changed considerably. The only notable warm and cold regions at $12 \mathrm{~h}$ are at the vertical boundaries of the heating/cooling regions, although they are still very small in magnitude. Figure 10c shows that significant vertical motions originate at the lateral boundaries of the heating/cooling regions and propagate both towards the center and into the environment. This behavior is because the heating/cooling functions are horizontally homogeneous
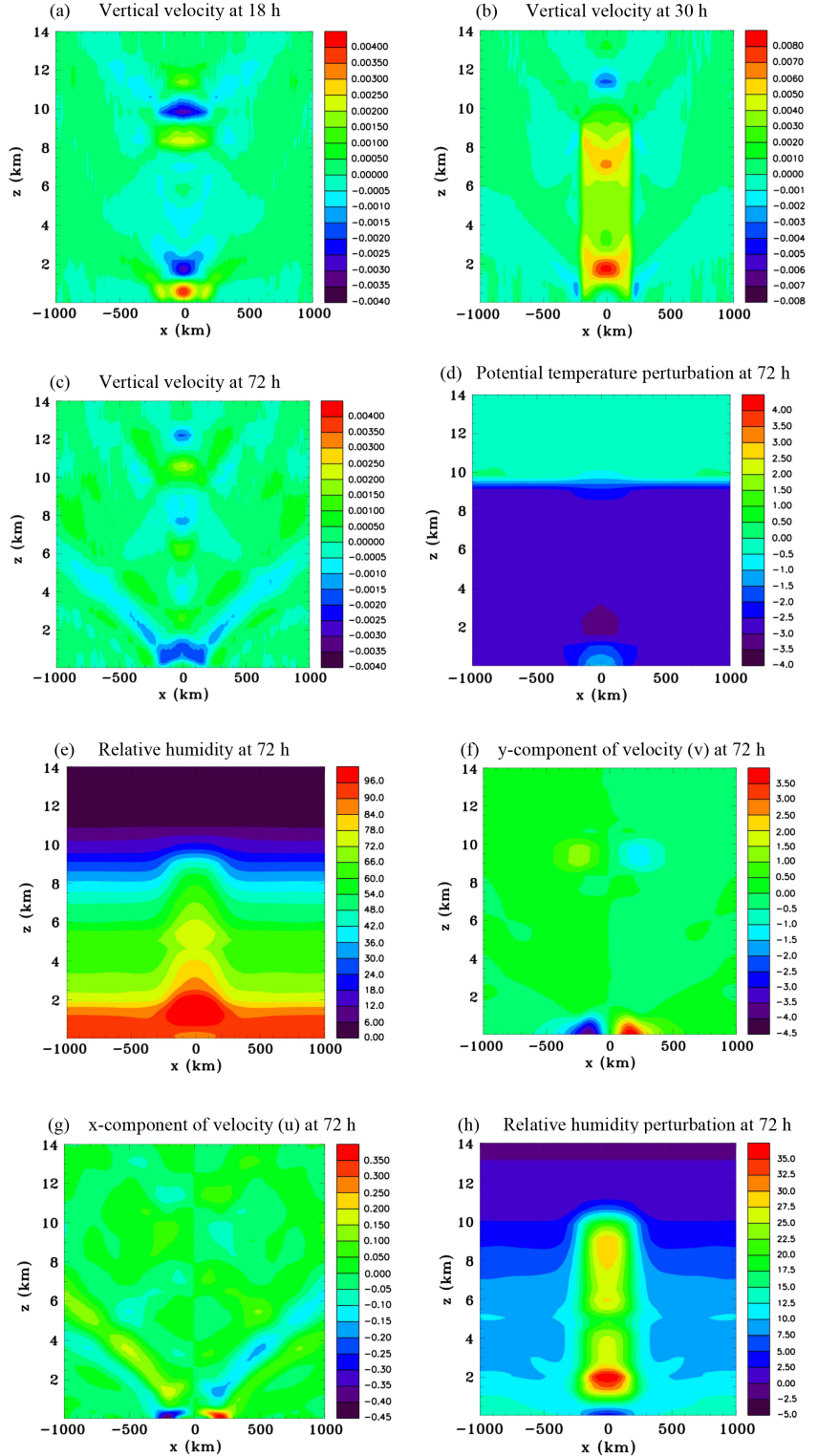

Figure 9. Vertical sections for Experiment 7: the idealized diurnal forcing case. (a) Vertical velocity at $t=18 \mathrm{~h}\left(\mathrm{~m} \mathrm{~s}^{-1}\right)$, (b) vertical velocity at $t=30 \mathrm{~h}\left(\mathrm{~m} \mathrm{~s}^{-1}\right),(\mathbf{c})$ vertical velocity at $t=72 \mathrm{~h}\left(\mathrm{~m} \mathrm{~s}^{-1}\right)$, (d) potential temperature perturbation at $t=72 \mathrm{~h}(\mathrm{~K})$, (e) relative humidity at $t=72 \mathrm{~h}$, (f) y-component of velocity, $v$ at $t=72 \mathrm{~h}$ $\left(\mathrm{m} \mathrm{s}^{-1}\right)$, (g) x-component of velocity, $u$ at $t=72 \mathrm{~h}\left(\mathrm{~m} \mathrm{~s}^{-1}\right)$, and (h) relative humidity perturbation at $t=72 \mathrm{~h}$.

except at the lateral boundaries. If the heating/cooling functions were strongly peaked at the center, then the largest magnitudes of vertical velocity would have started at the center. This situation is similar in some ways to a sea breeze circulation that begins at the land-sea boundary. Since the depth of the thermally driven circulations are shallower than for previous simulations, their lateral propagation is slower (Nicholls et al., 1991, and Eq. (1) of this paper). At $12 \mathrm{~h}$ there is broad 

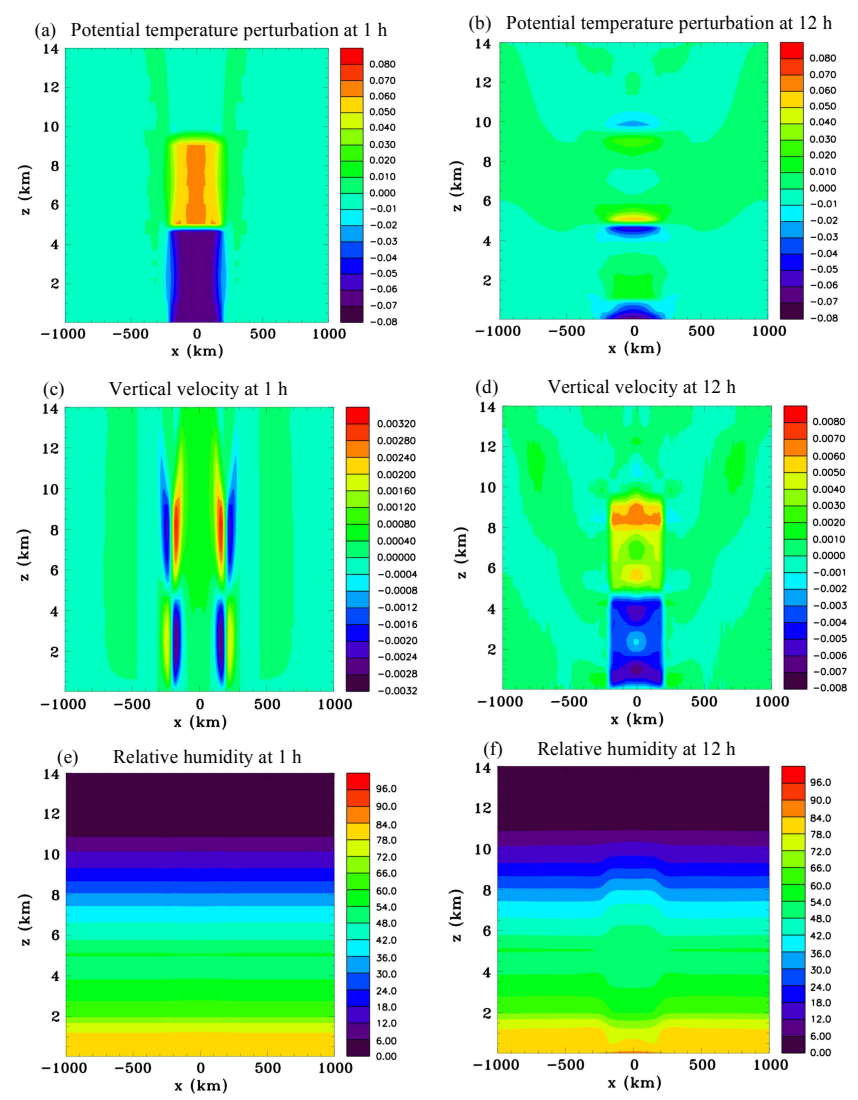

Figure 10. Vertical sections for Experiment 8: the forced core case at $t=1$ and $12 \mathrm{~h}$. (a) and (b) potential temperature perturbation (K), (c) and (d) vertical velocity $\left(\mathrm{m} \mathrm{s}^{-1}\right)$, and (e) and (f) relative humidity, at $t=1$ and $12 \mathrm{~h}$, respectively.

upward motion in the heated region and downward motion in the cooling region, although there are considerable inhomogeneities. This has led to a notable increase in relative humidity in the heating region and a decrease in the cooling region. One aspect that this experiment illustrates is that care has to be taken when inferring changes in static stability that might arise from radiative heating profiles that vary in the vertical, because in this case, where the forcing has limited horizontal extent, these changes become negligible in a short time.

Experiment 9 considers the response when there is a relatively weak vortex present. The radial distribution of uniform cooling $z<10 \mathrm{~km}$ is the same as in Experiment 3. The vortex has a depth of $12 \mathrm{~km}$ and is strongest at the surface, with a wind speed of $12 \mathrm{~m} \mathrm{~s}^{-1}$ at a radius of $75 \mathrm{~km}$. Figure 11a shows the initial relative humidity, which is slightly perturbed in the vortex because of the temperature and pressure anomalies associated with it, while Fig. 11b-e show vertical sections at $12 \mathrm{~h}$ of the $y$-component of velocity, potential temperature perturbation, vertical velocity and relative humidity, respectively. At $12 \mathrm{~h}$ there has been only a slight weakening of the vortex strength. The temperature has
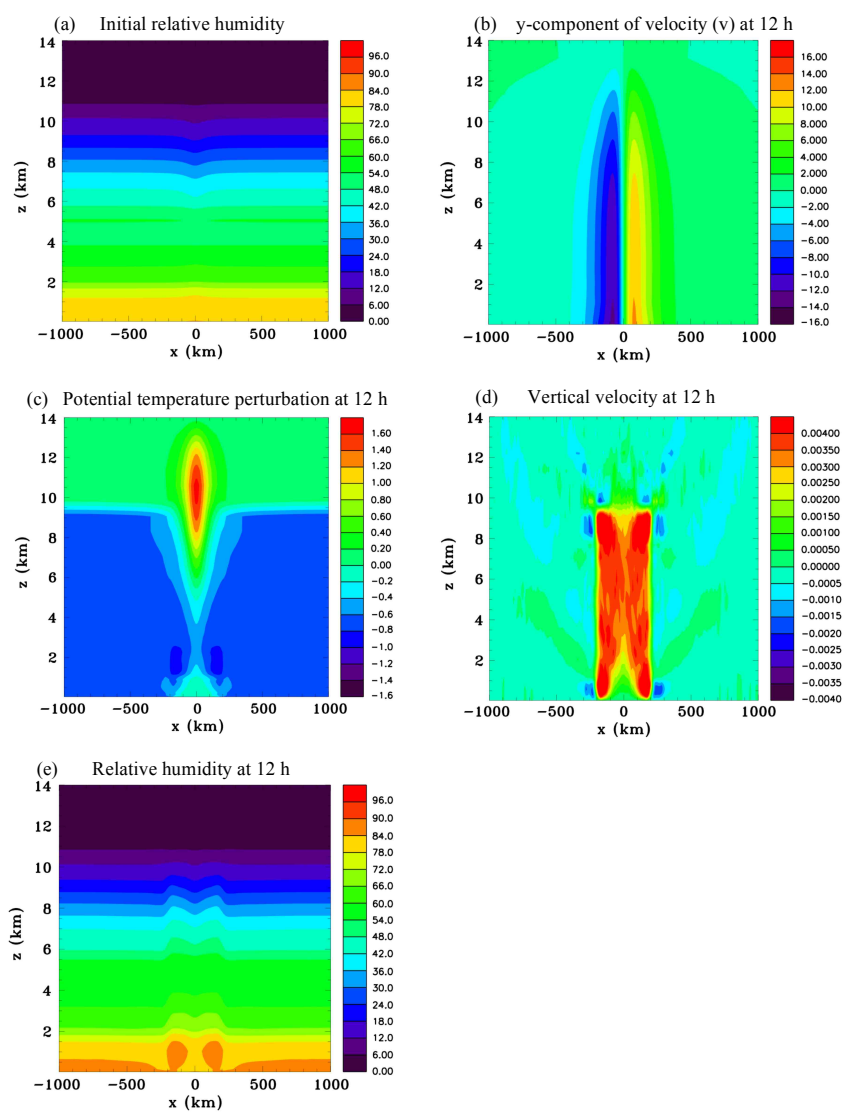

Figure 11. Vertical sections for Experiment 9: the weak vortex case. (a) Relative humidity at $t=0 \mathrm{~h}$, (b) $y$-component of velocity, $v$ at $t=12 \mathrm{~h}\left(\mathrm{~m} \mathrm{~s}^{-1}\right),(\mathbf{c})$ potential temperature perturbation at $t=12 \mathrm{~h}$

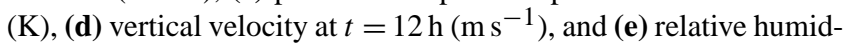
ity at $t=12 \mathrm{~h}$.

cooled significantly in the environment and throughout most of the vortex except near the surface central region where the vertical velocity is weak. The vertical velocity peaks just outside the radius of maximum winds, which leads to the most significant increase in relative humidity at this location. This result shows that the vortex is having a considerable influence on the induced motion in the unforced core.

Experiment 10 is similar to the previous experiment except that the vortex strength is increased to $30 \mathrm{~m} \mathrm{~s}^{-1}$. Figure 12 shows a more pronounced minimum in vertical velocity at the center of the vortex. Just outside the radius of maximum winds there is still a significant increase in the relative humidity. This result suggests that environmental radiative forcing at lower and middle levels could still have a significant influence on the convection within a strong tropical cyclone, but that it is more likely to impact the outer region of the system.

Several recent theoretical studies have investigated the response to heating in a tropical cyclone-like vortex under the assumption of gradient wind balance in the radial momentum 

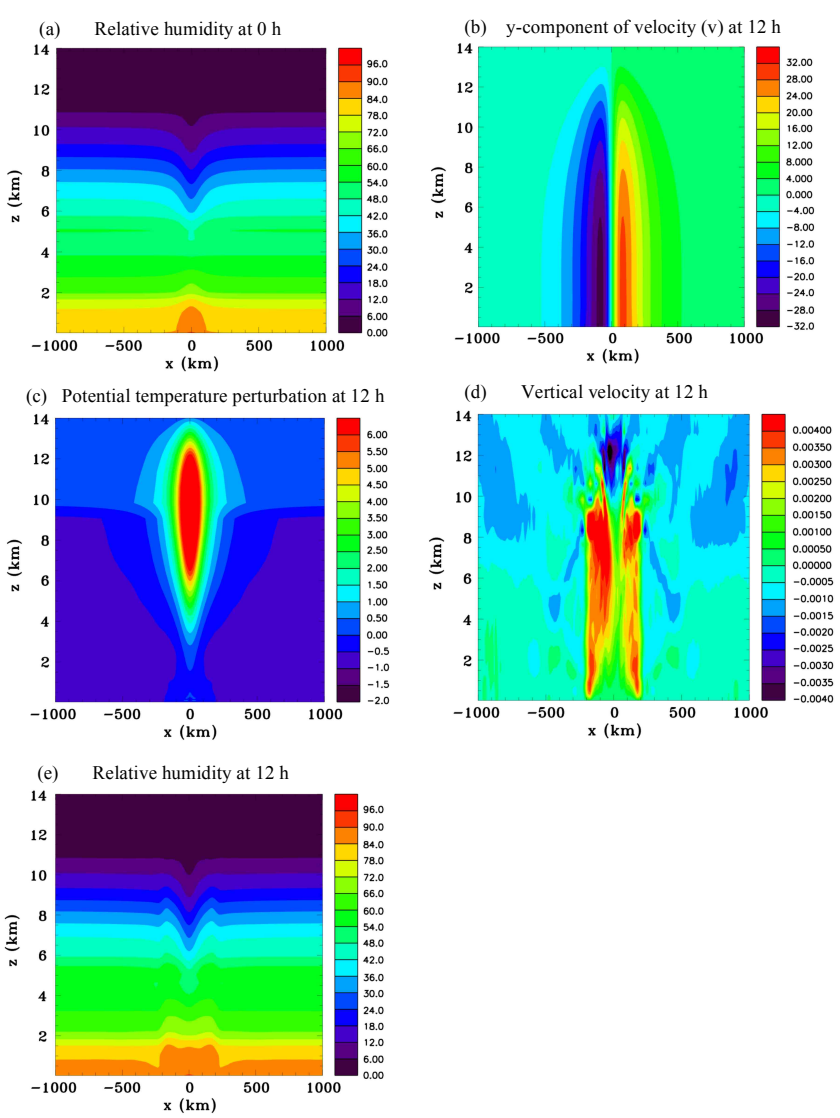

Figure 12. Vertical sections for Experiment 10, the strong vortex case. (a) Relative humidity at $t=0 \mathrm{~h}$, (b) $y$-component of velocity, $v$ at $t=12 \mathrm{~h}\left(\mathrm{~m} \mathrm{~s}^{-1}\right)$, (c) potential temperature perturbation at $t=12 \mathrm{~h}(\mathrm{~K}),(\mathbf{d})$ vertical velocity at $t=12 \mathrm{~h}\left(\mathrm{~m} \mathrm{~s}^{-1}\right)$, and (e) relative humidity at $t=12 \mathrm{~h}$.

equation (Wirth and Dunkerton, 2006, 2009; Pendergrass and Willoughby, 2009; Vigh and Schubert, 2009). These analyses lead to consideration of the "transverse circulation equation" first derived by Eliassen (1951). A similar analysis that includes representation of environmental radiative forcing could potentially provide a fuller interpretation of the results found for the vortex experiments shown in this study. The neglect of the time derivative of radial velocity in the radial momentum equation means that the thermally generated pulses of vertical motion that eventually propagate into the far environment would not be simulated; nevertheless the induced sustained upward motion in the vortex should qualitatively be the same. This could lend insight into the reason for the radial variation of vertical velocity induced in the vortex. A reasonable speculation is that it is a consequence of the inertial stability of the vortex.
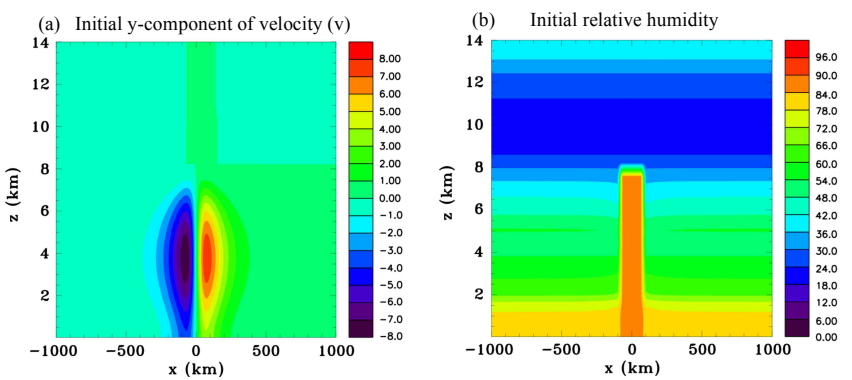

Figure 13. Vertical sections for Experiment 11: the radiation scheme activated in the whole domain with a mid-level vortex case. (a) $y$-component of velocity, $v\left(\mathrm{~m} \mathrm{~s}^{-1}\right)$, and (b) relative humidity, at $t=0 \mathrm{~h}$.

\subsection{Idealized experiments with radiation scheme included}

Experiment 11 proceeds to examine the response to the Harrington radiation scheme, instead of specified forcing, for the same initial vortex that will be used for the full physics simulations. There are no clouds or cloud-radiative feedbacks. Figure 13 shows vertical sections of the initial $y$-component of velocity and relative humidity. The core of the vortex has been moistened to $85 \%$ of saturation similarly to many of the experiments in NM13, which encourages the development of a tropical cyclone in the full physics simulations. A difference with the previous simulations is that instead of the vapor mixing ratio abruptly being set to zero above $11 \mathrm{~km}$ there is a more gradual decrease with height. The infrared cooling between 7 and $9 \mathrm{~km}$ is quite sensitive to the existence of small amounts of water vapor aloft. A more gradual reduction with height decreases the infrared cooling rate in this layer (Norman Wood, personal communication), which without this modification is quite large.

Figure 14 shows the radiative flux convergence and vertical velocity at $4 \mathrm{~h}$ into the simulation, which is during the middle of the day. At this time the solar heating is stronger than the infrared cooling in the upper troposphere and between 1 and $6 \mathrm{~km}$. The strongest cooling occurs at the top of the moistened core and the strongest heating between 5 and $6 \mathrm{~km}$, producing downward and upward motion, respectively. An east-west asymmetry can be seen in the radiative forcing due to longitudinal variation of the solar radiation.

Figure 15 shows fields at $10 \mathrm{~h}$ during the early nighttime. A layer of strong cooling of approximately $-3 \mathrm{~K}$ day $^{-1}$ occurs outside the moistened core between $z=7$ and $8 \mathrm{~km}$. An even stronger cooling in this layer occurs in the moistened core. There is a maximum near $4 \mathrm{~km}$ and moderate cooling below in the environment. There is a maximum at low levels in the moistened core. At this time the only significant vertical velocity is downward at the top of the moistened core where the cooling is strongest. There has not been a significant change to the relative humidity from the initial values. 

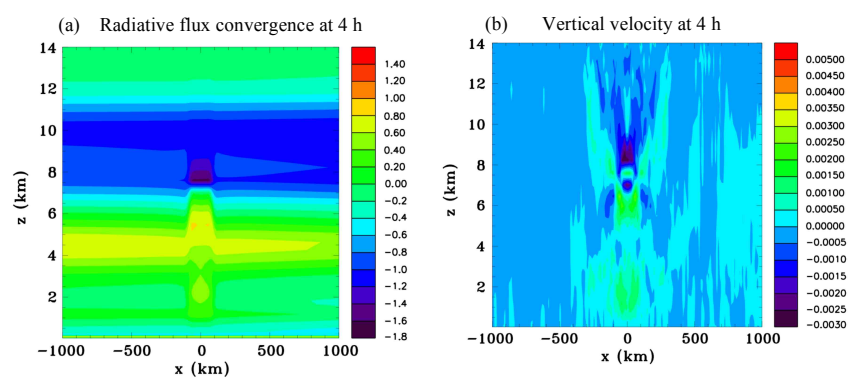

Figure 14. Vertical sections for Experiment 11: the radiation scheme activated in the whole domain with a mid-level vortex case. (a) Radiative flux convergence $\left(\mathrm{K} \mathrm{s}^{-1} \times 10^{-5}\right)$, and (b) vertical velocity $\left(\mathrm{m} \mathrm{s}^{-1}\right)$, at $t=4 \mathrm{~h}$, during the day.

The strong environmental cooling produced by the Harrington radiation scheme at around the $8 \mathrm{~km}$ level is similar to the result found for infrared cooling during moist conditions in the equatorial western Pacific by Zhang and Chou (1999), who calculated a maximum cooling at around $300 \mathrm{mb}$ (Fig. 4 of their paper).

Figure 16 shows fields at $24 \mathrm{~h}$, which is mid-morning. The potential temperature perturbation is negative in the environment throughout the troposphere except at the very top. Beneath $3.5 \mathrm{~km}$ there has been a cooling of approximately -1.5 $\mathrm{K}$. Between 4 and $5 \mathrm{~km}$ it is about half as large, whereas it is more than $-2 \mathrm{~K}$ day $^{-1}$ between 7 and $8 \mathrm{~km}$. The overall cooling has led to a significant increase in relative humidity. Note that the contour intervals in Fig. 16c have been changed from previous figures for better comparison with the next experiment. Subsidence is still noticeable at the top of the vortex at $24 \mathrm{~h}$ and weak upward motion above. The vortex winds have not changed a great deal in the $24 \mathrm{~h}$ period, showing only a slight weakening, probably due to model diffusion.

Experiment 12 is similar to the previous one, except the radiation is turned off for radius less than $200 \mathrm{~km}$. Figure 17 shows fields at $4 \mathrm{~h}$. The radiative flux convergence in Fig. 17a is identical to that in Fig. 14a for radius greater than $200 \mathrm{~km}$. A significant upward motion is induced in the core within the 7-12 km layer, where there is cooling in the environment. Conversely downward motion is induced in the core within the 3-6 km layer where there is warming in the environment.

Figure 18 shows that at $10 \mathrm{~h}$ during the nighttime there is significant upward motion at upper levels associated with the strong environmental cooling aloft. The sustained upward motion both during the day and night has led to increased humidity in the core at upper levels. The perturbed relative humidity field also shows that, even though a nested grid was used to increase horizontal resolution, there is still some noticeable lateral diffusion due to the subgrid-scale turbulence scheme where the velocity gradients are large.

Figure 19 shows fields at $24 \mathrm{~h}$. There has been a more significant reduction in potential temperature in the warm core aloft compared to Experiment 11 (Fig. 16a). Also, the air at
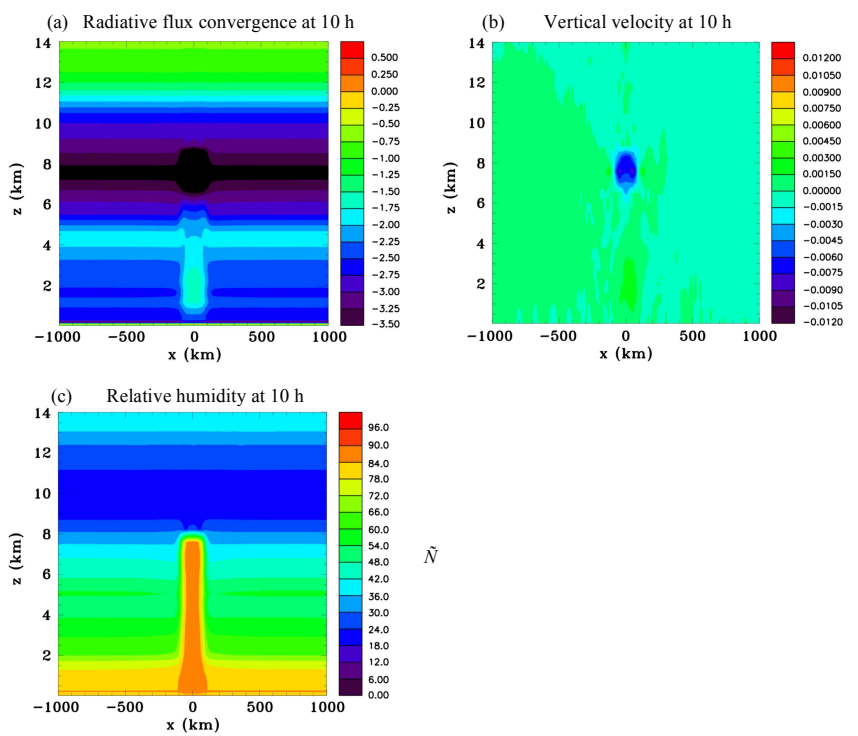

Figure 15. Vertical sections for Experiment 11: the radiation scheme activated in the whole domain with a mid-level vortex case. (a) Radiative flux convergence $\left(\mathrm{K} \mathrm{s}^{-1} \times 10^{-5}\right)$, (b) vertical velocity $\left(\mathrm{m} \mathrm{s}^{-1}\right.$ ), (c) relative humidity, at $t=10 \mathrm{~h}$, during the night.

the surface in the core is significantly warmer than the environmental air. At this time the strongest upward motion is near the surface at a radius of $200 \mathrm{~km}$. There has been a large increase in relative humidity in the core to values far in excess of saturation at a height of $8 \mathrm{~km}$. The larger increase in relative humidity aloft compared to low levels for this simulation that uses the radiation scheme is in contrast to the results of Experiments 2 and 7 that showed largest increases at low levels. Note that since the model does not have microphysics activated the accumulation of moisture to values well in excess of saturation is able to occur. Another difference with the previous experiment is that wind speeds increase at the surface by approximately $1.5 \mathrm{~m} \mathrm{~s}^{-1}$.

The results of Experiment 11 during the first $12 \mathrm{~h}$ are similar to what occurs in the full physics simulation with radiation activated, which will be discussed shortly. There are some differences since the full physics simulation has surface fluxes, and, also, shallow clouds develop by $12 \mathrm{~h}$. There is a small but still significant increase in low-level relative humidity both in the core and in the environment in Experiment 11 , which in the full physics simulation tends to promote the development of low-level clouds. In the full physics simulation with radiation included in the whole domain, deep convection develops after $12 \mathrm{~h}$, which shortly thereafter causes an upper-level canopy to form that modifies the radiative fluxes considerably. It will be shown that this results in a reduction of longwave cooling at low levels in the core and a slight warming at middle levels. Experiment 12 is idealized since the radiative forcing is set to zero in the core beneath $10 \mathrm{~km}$, but does illustrate that weak but still significant circulations could be expected to develop in the more 

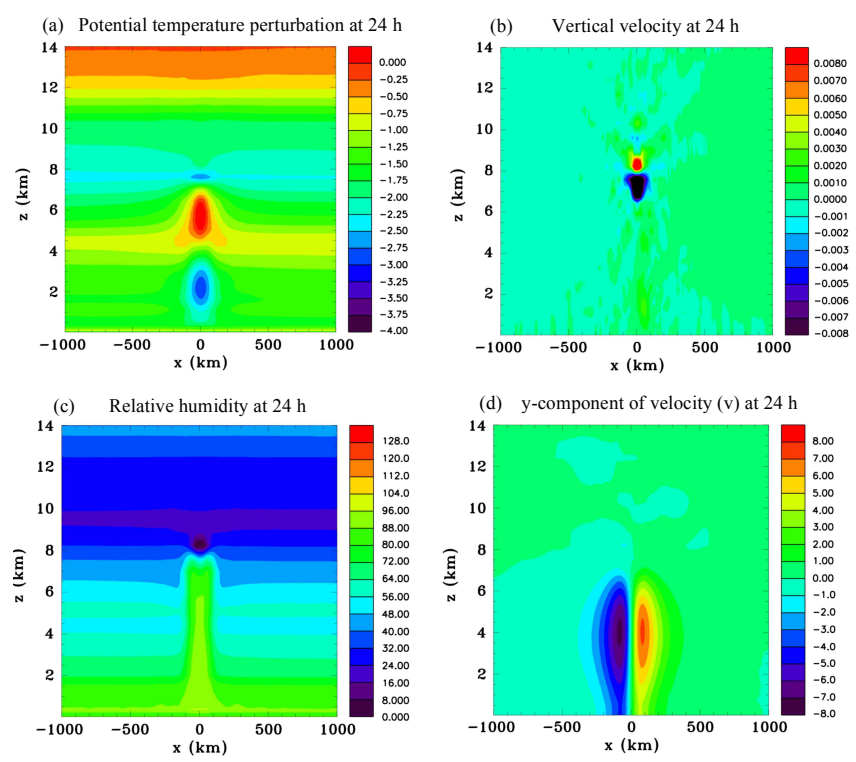

Figure 16. Vertical sections for Experiment 11: the radiation scheme activated in the whole domain with a mid-level vortex case. (a) Potential temperature perturbation $(\mathrm{K})$, (b) vertical velocity $\left(\mathrm{m} \mathrm{s}^{-1}\right)$, (c) relative humidity, and (d) $y$-component of velocity, $v\left(\mathrm{~m} \mathrm{~s}^{-1}\right)$, at $t=24 \mathrm{~h}$, in mid-morning.
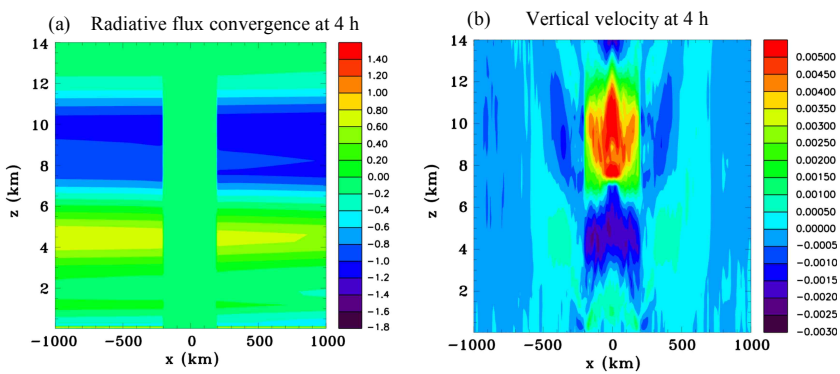

Figure 17. Vertical sections for Experiment 12: the radiation scheme activated in the environment, $r>200 \mathrm{~km}$. (a) Radiative flux convergence $\left(\mathrm{K} \mathrm{s}^{-1} \times 10^{-5}\right)$, and (b) vertical velocity $\left(\mathrm{m} \mathrm{s}^{-1}\right)$, at $t=4 \mathrm{~h}$.

complex full physics simulations associated with the differential radiative forcing between the environment and the core when a cloud canopy forms.

\subsection{Full physics experiments}

The next set of experiments to be discussed have surface fluxes and cloud microphysics. Experiment 13 that has radiation included will be described in some detail. Figure 20 shows a horizontal section of total hydrometeor mixing ratio at a height of $11.7 \mathrm{~km}$ at $15 \mathrm{~h}$, and vertical sections through the center of the domain of total hydrometeor mixing ratio and radiative flux convergence at $21 \mathrm{~h}$. Figure 20a shows that several deep moist convective cells have developed by $15 \mathrm{~h}$ and a canopy aloft is starting to form as the anvils from the
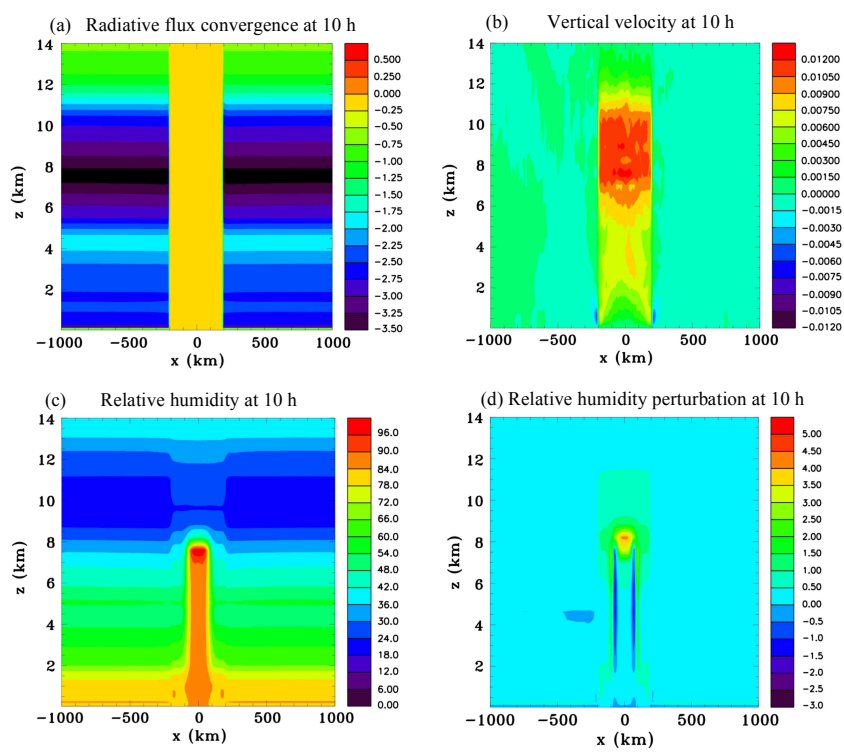

Figure 18. Vertical sections for Experiment 12: the radiation scheme activated in the environment, $r>200 \mathrm{~km}$. (a) Radiative flux convergence $\left(\mathrm{K} \mathrm{s}^{-1} \times 10^{-5}\right)$, (b) vertical velocity $\left(\mathrm{m} \mathrm{s}^{-1}\right)$, (c) relative humidity, and (d) relative humidity perturbation, at $t=10 \mathrm{~h}$.
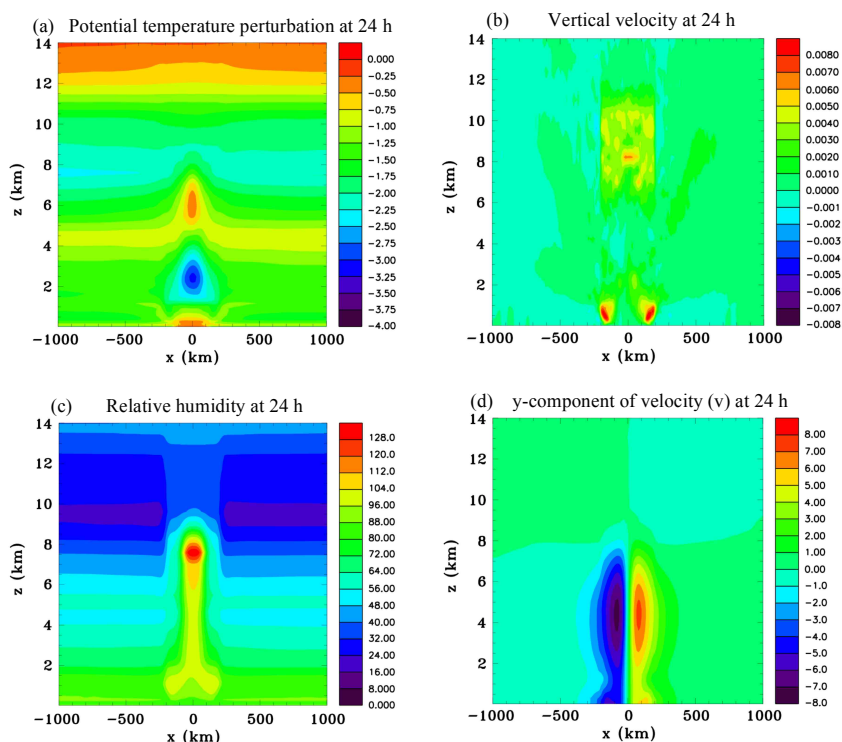

Figure 19. Vertical sections for Experiment 12: the radiation scheme activated in the environment, $r>200 \mathrm{~km}$. (a) Potential temperature perturbation $(\mathrm{K}),(\mathbf{b})$ vertical velocity $\left(\mathrm{m} \mathrm{s}^{-1}\right)$, (c) relative humidity, and (d) $y$-component of velocity, $v\left(\mathrm{~m} \mathrm{~s}^{-1}\right)$, at $t=24 \mathrm{~h}$.

individual cells begin to merge. Figure 20b shows that $6 \mathrm{~h}$ later the canopy aloft is more widespread. A strong cell is evident at $x=90 \mathrm{~km}$. Figure 20c shows that at this time, which is during the night just before daybreak, there is strong cooling at the top of the canopy. There is also strong warming at the base of the canopy in the outer region of the core. 

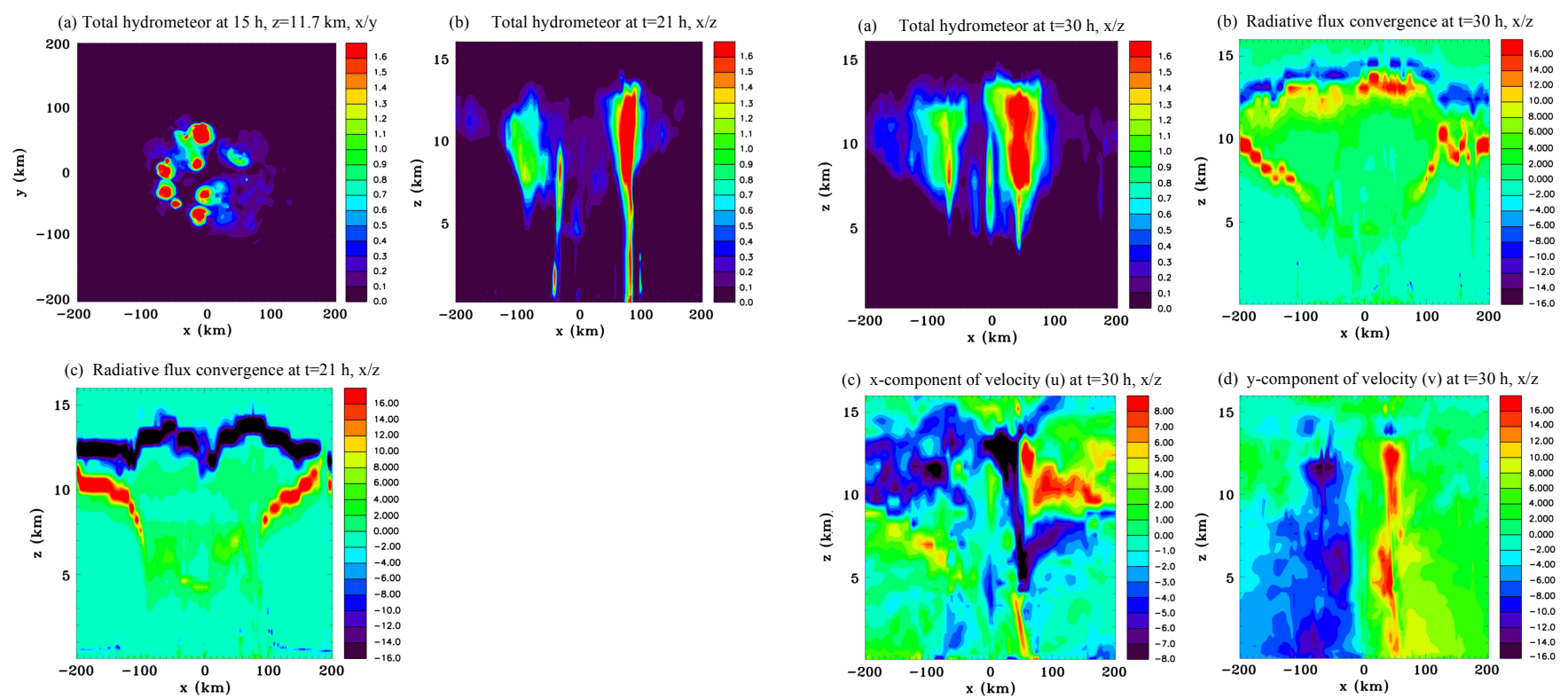

Figure 20. Experiment 13: full physics with radiation in the whole domain. (a) Horizontal section of total hydrometeor mixing ratio at $z=11.7 \mathrm{~km} . t=15 \mathrm{~h}\left(\mathrm{~g} \mathrm{~kg}^{-1}\right)$, (b) vertical section of total hydrometeor mixing ratio at $t=21 \mathrm{~h}\left(\mathrm{~g} \mathrm{~kg}^{-1}\right)$, and (c) vertical section of radiative flux convergence at $t=21 \mathrm{~h}\left(\times 10^{-5} \mathrm{~K} \mathrm{~s}^{-1}\right)$.

Figure 21 shows vertical sections at $30 \mathrm{~h}$, which is in the late afternoon. The cloud canopy has continued to develop. There is significant shortwave warming of the cloud canopy aloft, but longwave cooling at the top of the cloud canopy is also starting to occur by this time. It is notable that the shortwave warming of the cloud canopy aloft occurs just beneath the region of longwave cooling. The $x$-component of velocity shown in Fig. 21c has strong velocities associated with the intense cell present at $x=50 \mathrm{~km}$. There is also a sloping inflow evident at the base of the cloud layer, which is very persistent and which leads to the spinup of the mid-level circulation seen to be occurring in Fig. 21d, between $z=4$ and $7 \mathrm{~km}$. The mid-level circulation continued to intensify and at $38 \mathrm{~h}$ a small vortex suddenly formed, which was concentrated at the surface and close to the center of the domain.

Figure 22 shows a horizontal section of the vertical relative vorticity near the surface at $40 \mathrm{~h}$ and a vertical section of the $y$-component of velocity at $48 \mathrm{~h}$. A small region of intense low-level positive vertical vorticity can be seen at the center of the domain at $40 \mathrm{~h}$. This small vortex became the focus of a rapidly intensifying circulation and cyclonic winds exceeded $30 \mathrm{~m} \mathrm{~s}^{-1}$ by $48 \mathrm{~h}$. The development of this tropical cyclone was along pathway Two as discussed in NM13, and appears to be similar to the results of Nolan (2007). The evolution was similar to other cases discussed in NM13 that evolved along pathway Two, even though there are some differences in the model parameterizations and in the initial moisture profile, as discussed in Sect. 2. The tropical cyclone

Figure 21. Experiment 13: full physics with radiation in the whole domain. Vertical sections at $t=30 \mathrm{~h}$ of (a) the total hydrometeor mixing ratio $\left(\mathrm{g} \mathrm{kg}^{-1}\right)$, (b) radiative flux convergence at $t=21 \mathrm{~h}$ $\left(\times 10^{-5} \mathrm{~K} \mathrm{~s}^{-1}\right)$, (c) $x$-component of velocity, $u\left(\mathrm{~m} \mathrm{~s}^{-1}\right)$, and (d) $y$-component of velocity, $v\left(\mathrm{~m} \mathrm{~s}^{-1}\right)$.
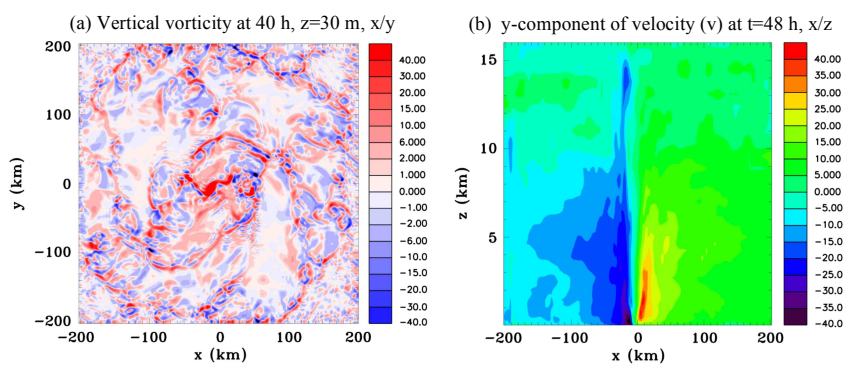

Figure 22. Experiment 13: full physics with radiation in the whole domain. (a) Horizontal section of surface vertical vorticity, at $t=40 \mathrm{~h}\left(\times 10^{-4} \mathrm{rad} \mathrm{s}^{-1}\right)$, and (b) vertical section of the $y$ component of velocity, $v$, at $48 \mathrm{~h}\left(\mathrm{~m} \mathrm{~s}^{-1}\right)$.

that formed is small and compact, which is an advantage for these experiments since it does not require a large fine-scale grid.

Figure 23 shows time series of the maximum near-surface azimuthally averaged tangential wind speeds and total hydrometeor mass in the domain for the first five experiments shown in Table 4. Also shown is the shortwave cloud-free radiation reaching the surface for a simulation without microphysics, initial vortex or surface fluxes. Experiment 13 with radiation throughout the whole domain intensifies very similarly to Experiment 15 with radiation only in the environment (radius greater than $200 \mathrm{~km}$ ). Near-surface wind speeds reached $12 \mathrm{~m} \mathrm{~s}^{-1}$ around $36 \mathrm{~h}$ followed by a rapid rate of intensification until $48 \mathrm{~h}$ and then a somewhat slower rate until 
the end of the simulation at $84 \mathrm{~h}$. The fastest development occurred for Experiment 17 with environmental cooling beneath a height of $10 \mathrm{~km}$ at a constant rate of $-1.5 \mathrm{~K} \mathrm{day}^{-1}$. The case without radiation or any prescribed forcing did not start to intensify until the very end of the simulation. The case with radiation only in the core also did not develop until late in the simulation, but did show a very rapid intensification beginning at $72 \mathrm{~h}$. These results suggest that the radiation in the environment is an important factor in causing the accelerated rate of development when radiation is activated in the model. Including radiation only in the core dramatically slowed the development compared with including radiation everywhere. Therefore, it appears unlikely that the very strong upper-level radiative heating and cooling rates in the core of the system are having a large effect on the rate of development. It seems more likely that the circulation induced by differential heating and cooling between the environment and the cloudy region at low and middle levels is the main cause of the accelerated development when radiation is included. The previous idealized experiments suggest that an increase in relative humidity due to weak upward motion in the core, particularly significant during the nighttime when environmental cooling is strongest, is the main factor responsible.

The total hydrometeor mass in the fine grid domain in Fig. 23b shows a very significant oscillation between 36 and $48 \mathrm{~h}$ occurring for both the case with radiation included everywhere and for the case with radiation only included in the environment (Experiments 13 and 15). Figure 23c shows that this coincides with the second night and that the rapid increase in total hydrometeor mass came to an end during the early daylight hours. The case with radiation included only in the environment also had a significant oscillation that began during the first night. In NM13, diurnal oscillations of the total hydrometeor mass also occurred for simulations with radiation included, a particularly prominent example being shown in Fig. 11 of that paper. It can also be seen that Experiment 17 with thermal forcing constant in time also had two moderately large oscillations early on.

In order to obtain a better understanding of the role of radiative forcing, time and azimuthal averages of the radiative flux convergence were made for Experiment 13 and are shown in Fig. 24. Figure 24a shows an average between 24 and $48 \mathrm{~h}$ for the whole troposphere, whereas Fig. 24b shows the same cross section magnified for the lower troposphere. Figure $24 \mathrm{c}$ shows a $6 \mathrm{~h}$ average for the previous night between 15 and $21 \mathrm{~h}$, and Fig. 24d shows a $6 \mathrm{~h}$ average in the daytime between 24 and $30 \mathrm{~h}$. Figure 24a is dominated by the strong radiative forcing at upper levels. The warming at the base of the stratiform ice layer is quite smeared due to its variation over this $24 \mathrm{~h}$ period. Figure $24 \mathrm{~b}$, which provides more detail of the lower and middle troposphere, shows significant horizontal gradients of the $24 \mathrm{~h}$ averaged radiative forcing. There is a slight cooling at low levels and a slight warming at middle levels in the core. The horizon-
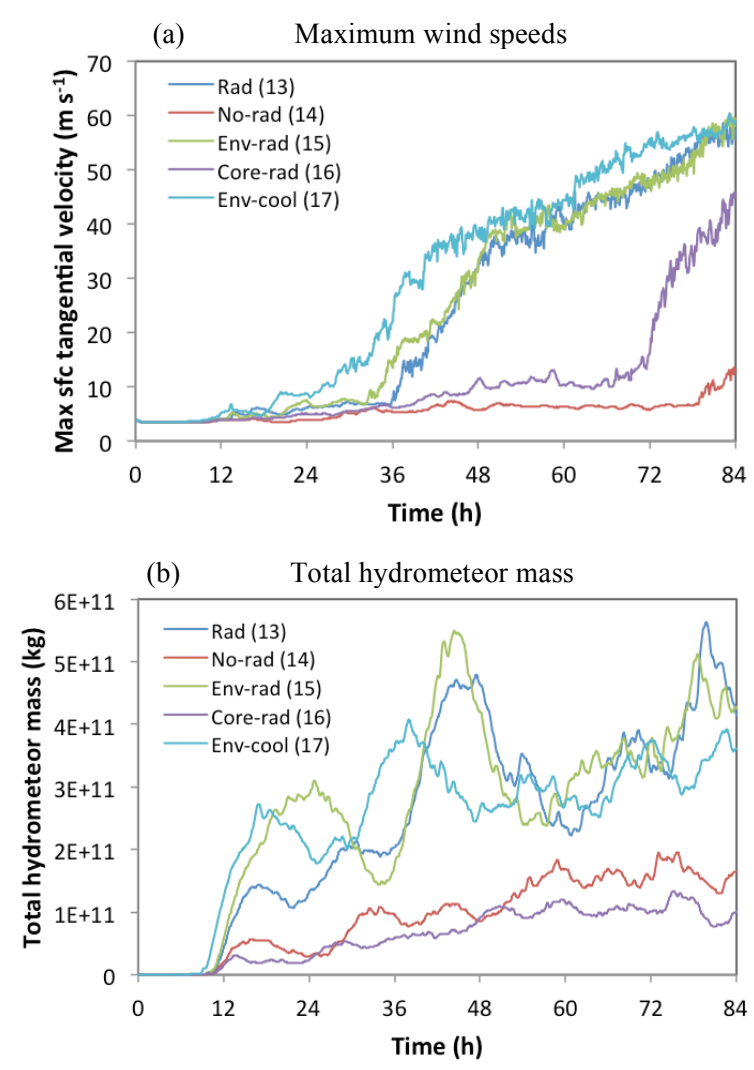

(c) Clear-sky short wave radiation

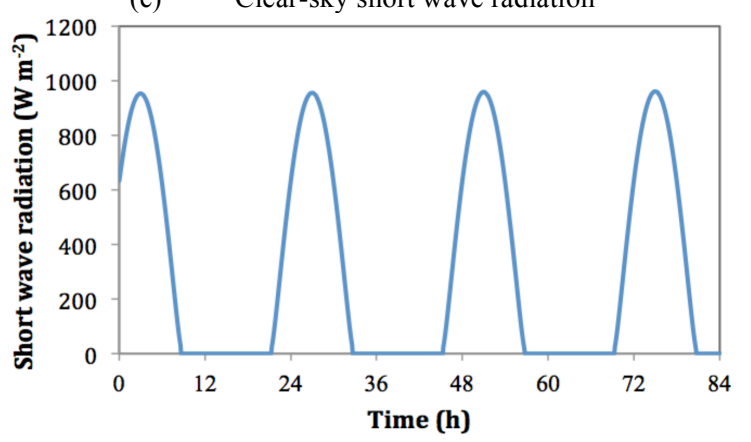

Figure 23. Time series for Experiments 13-17: (a) maximum azimuthally averaged tangential wind speeds at $z=29.5 \mathrm{~m}\left(\mathrm{~m} \mathrm{~s}^{-1}\right)$, (b) total hydrometeor mass in the domain $(\mathrm{kg})$, and (c) clear-sky short wave radiation at the surface $\left(\mathrm{W} \mathrm{m}^{-1}\right)$.

tal difference between the core and the environment is about $-1 \mathrm{~K} \mathrm{day}^{-1}$ at low levels and slightly larger in magnitude at mid levels. During this period a shallow cloud layer developed, which extended into the environment, and there is quite strong longwave cooling that can be seen at the top of this layer. Figure $24 \mathrm{c}$ shows a pronounced horizontal gradient during the previous nighttime that is particularly large aloft. In the daytime Fig. 24d shows that the gradients are a lot weaker and at low levels reversed. Based on the idealized simulations discussed previously, the magnitude of the averaged horizontal gradients at low and mid levels appear 

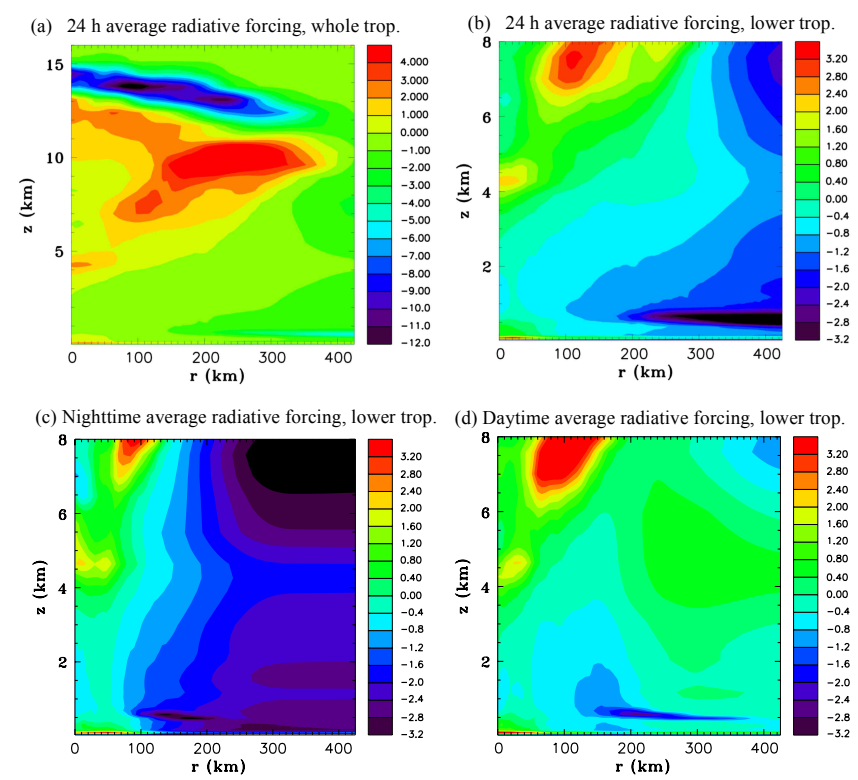

Figure 24. Time and azimuthally averaged radiative flux convergence for Experiment 13: the full physics simulation with radiation activated in the whole domain case. (a) $24 \mathrm{~h}$ average from 24 to $48 \mathrm{~h}$ shown for the whole troposphere, (b) $24 \mathrm{~h}$ average from 24 to $48 \mathrm{~h}$ shown for the lower troposphere, (c) $6 \mathrm{~h}$ nighttime average from 15 to $21 \mathrm{~h}$ shown for the lower troposphere, and (d) $6 \mathrm{~h}$ daytime average from 24 to $30 \mathrm{~h}$ shown for the lower troposphere $\left(\times 10^{-5} \mathrm{~K} \mathrm{~s}^{-1}\right)$.

large enough to drive circulations, resulting in significant changes in the core capable of promoting convective activity. Since the gradients of radiative forcing are stronger aloft, the largest increase in relative humidity in the core is also likely to be aloft. This also applies to cooling, which would tend to make convective updrafts stronger at upper levels. For this environment and these particular RAMS simulations a tentative conclusion is that the radiative forcing is making the mid-level local environment more favorable for enhancing convection that has been triggered at low levels, and this could be more important than causing an increase in the lowlevel convective triggering, although both are likely occurring.

Experiments 18-24 were conducted in order to obtain a clearer picture of the role of radiative forcing suggested by the foregoing results. For instance, Experiment 16 with radiation included only for radius less than $200 \mathrm{~km}$ and which developed slowly seems to suggest that mean cooling of the environment is a major factor responsible for the accelerated rate of genesis. Presumably if the radius within which radiation is included were increased say to $400 \mathrm{~km}$, this would not be large enough to encompass much of the environment surrounding the cloud disturbance and therefore genesis would remain slow. Therefore Experiment 18 includes radiation for radius less than $400 \mathrm{~km}$. It also might be expected that if radiation were included only in the environment but for a larger radius than $200 \mathrm{~km}$, which was the value used in Ex-

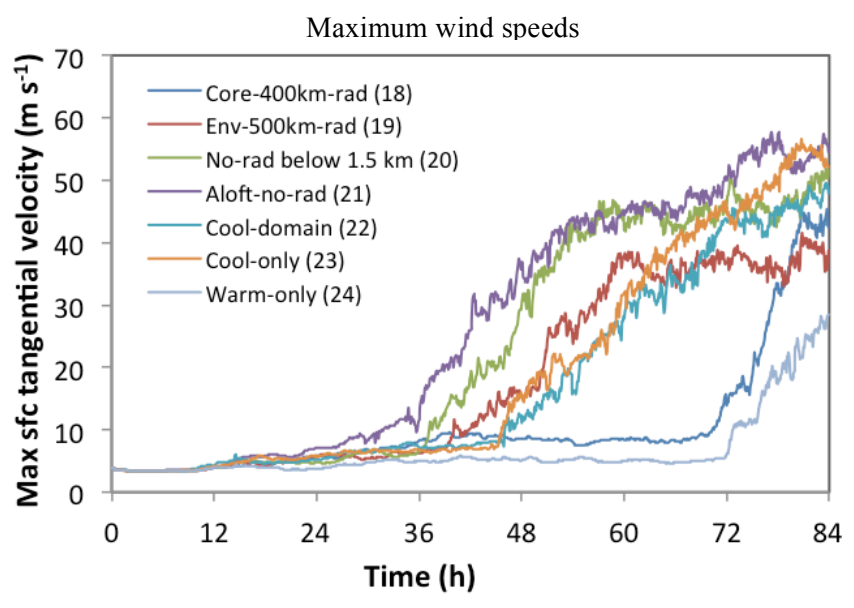

Figure 25. Time series of maximum azimuthally averaged tangen-

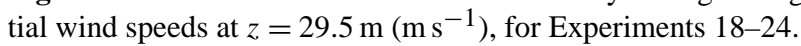

periment 15 , then there would be an accelerated rate of genesis, but probably not as rapid as for Experiment 15. Therefore Experiment 19 includes radiation for radius greater than $500 \mathrm{~km}$. An interesting issue is how important radiative forcing at low levels is for increasing the rate of genesis when radiation is included. To examine this issue Experiment 20 has radiation included except in the lowest $1.5 \mathrm{~km}$. Equally interesting is the importance of radiation at upper levels for increasing the genesis rate. Therefore Experiment 21 has radiation turned off above $8 \mathrm{~km}$ to see how this affects the rate of development. It has been noted in this and other studies that clear-sky radiative cooling of the atmosphere will produce increased relative humidity, which might be expected to promote convective development. Experiment 22 examines the effect of persistent horizontally uniform cooling to examine how the resultant increase in relative humidity affects convective development. For this case the radiation scheme is turned off but a uniform cooling rate of $-1.5 \mathrm{~K} \mathrm{day}^{-1}$ is prescribed beneath a height of $10 \mathrm{~km}$, throughout the domain. Finally, two more experiments are conducted, one with only radiative cooling (Experiment 23), and one with only radiative warming (Experiment 24). Combined with the results of other experiments these give a better indication of the relative importance of environmental radiative cooling versus mid-level radiative warming in the core in enhancing the development of the tropical disturbance.

Figure 25 shows the maximum near-surface azimuthally averaged tangential winds for Experiments 18-24. As expected, Experiment 18, which has a $400 \mathrm{~km}$ core region where radiation is included, has a slow genesis rate. The development was however quite complex. As a large stratiform anvil formed, extending out to approximately $200 \mathrm{~km}$ radius, there was still a considerable cloud-free area beyond it where the radiative transfer scheme likewise was activated. In this clear region surrounding the cloudy core, subsidence predominantly due to nighttime cooling produced a region 
of less humid air surrounding the developing cloud system. This scenario has similarities to Experiment 4 that examined the response to a small annulus of cooling between 200 and $400 \mathrm{~km}$. The cyclonic low-level wind speeds increased fairly significantly early on, more so than for Experiment 16 that had a $200 \mathrm{~km}$ core where radiation was included. This could have been partly due to upward motion induced by the surrounding subsidence, although Experiment 4 suggests that this might not be very large. Another factor is that the system was notably more compact, apparently because the subsiding ring of air was less humid, thereby reducing convective activity on the periphery of the cloud system. The more centrally focused convection may possibly have played a role in the early strengthening of the low-level circulation. In the long term the dry surrounding ring of air is likely to have been a factor inhibiting convection until the stratiform anvil grew large enough to reduce the cloud-free radiative forcing. The lack of mean cooling in the environment beyond a radius of $400 \mathrm{~km}$ also appears to have been a major factor in the slow genesis rate, as expected.

Experiment 19, with radiation included beyond a radius of $500 \mathrm{~km}$, developed a tropical cyclone more slowly than Experiment 15, with radiation beyond a radius of $200 \mathrm{~km}$ (Fig. 23a). Development was still however considerably faster than the case without radiation. This supports the conclusion that mean cooling in the large-scale surrounding environment is a major factor responsible for increasing the rate of tropical cyclogenesis in the model when radiation is included.

The case with no radiation beneath $1.5 \mathrm{~km}$ developed slightly slower than the case with radiation everywhere (Fig. 23a). This result indicates that the effects of radiative forcing above $1.5 \mathrm{~km}$ are having the most influence on the genesis rate.

Experiment 21 with no radiative forcing above $8 \mathrm{~km}$ developed the fastest among these seven experiments. The rate of development was similar to Experiment 13, with radiation at all levels. This result suggests that radiative forcings at lower and middle levels have the most impact on the genesis rate, in spite of there being much stronger heating and cooling rates aloft.

Experiment 22 with a prescribed horizontally uniform cooling beneath $10 \mathrm{~km}$ (including the core inside $200 \mathrm{~km}$ ) developed considerably slower than most of the other cases, but still significantly faster than the case without radiation. This supports the idea that increases in relative humidity due to large-scale radiative cooling without horizontal gradients can promote convective activity (e.g., Dudhia, 1989; Tao, 1996). However, it is apparently not as effective in promoting convective activity as differential horizontal radiative forcing, which generates circulations in the core. Moreover, the experiment is very idealized since once a cloud canopy develops it will likely cause differential horizontal forcing. As discussed in the introduction the question becomes the following: how influential is the increase in relative humidity in the cloud-free environment where cooling occurs? Another factor worth mentioning is that because there continues to be some weak longwave cooling at low levels in the simulated cloud system, which would tend to increase the humidity in situ, this could enhance convective activity to some extent.

Experiment 23 with negative radiative forcing only underwent genesis fairly quickly, but still significantly slower than with the full radiative forcing. Experiment 24 with positive radiative forcing only showed the slowest development in Fig. 25, but still faster than the no-radiation case (Experiment 19, Fig. 23a). Therefore, it appears that warming, probably at mid levels in light of Experiment 21, contributes to increasing the rate of genesis, but it is not as important as the large-scale environmental cooling in inducing a circulation in the core.

\section{Conclusions}

The results of this numerical modeling study suggest that circulations generated by differential radiative forcing may increase significantly the rate of tropical cyclogenesis and also play a major role in diurnal oscillations of convective activity. Experiments show that the influences of the radiatively induced circulations on convective activity in the system include (1) increased relative humidity, (2) changes in static stability, and (3) increased low-level cyclonic circulation. The first and second of these differential radiative forcing effects are caused by upward motion within the core, which is strongest during the night. The model results indicate that relatively weak vertical motions are induced in the core during the day, so that in the mean there is a net upward motion tendency caused by differential radiative forcing. The third influence is due to convergence of absolute vorticity by the low-level inflow, which results in a small increase in the low-level cyclonic winds. This is also likely to increase the rate of genesis.

It has not been shown explicitly in this study that vertical motions induced by differential radiative forcing are predominantly the cause of the accelerated rates of genesis in the full physics simulations when radiation is included, but nevertheless it is a strong inference that can be made. The radiatively induced vertical motions are very small compared to those produced by latent heating in convective towers, so it is very hard to discern them explicitly in a full physics simulation. It is assumed here that the small vertical velocity tendencies that would be expected due to the differential radiative forcing are superimposed on the stronger vertical motion produced in convective towers and in the regions between them where often there is also relatively strong vertical motion, for instance in stratiform cloud layers, shallow cumulus clouds, and in turbulence and gravity waves. This has not been demonstrated explicitly, but the fact that idealized simulations with prescribed differential forcing show significant impacts in the core, and that the same differential 
forcing applied in full physics simulations shows increased convective activity and faster rates of genesis, supports this contention.

This study interprets this problem in terms of the propagation of thermally generated gravity waves, or buoyancy bores. It was noted that a deep prescribed radiative cooling in a finite radial range produces deep rapidly propagating wave-like pulses of vertical motion in line with previous studies that have focused on their generation by latent heat release in convective systems. Observational studies by Bryan and Parker (2010) and Adams-Selin and Johnson $(2010,2013)$ appear to have detected the passage of this kind of gravity wave mode generated by convection in intense mesoscale systems. Modulations of radiative forcing in the real atmosphere are unlikely however to be large enough in magnitude, or rapid enough, to ever produce observable gravity wave modes of this type. The numerical results however strongly suggest that they are present. The result for the idealized diurnal forcing experiment, that the potential temperature changes at mid levels in the core closely parallel those in the environment with hardly any noticeable delay, are evidence of the fast propagation speed of these waves. Examination of the propagation of these waves also helped to explain the results of the annulus and forced core experiments. Although the inward propagating thermally induced circulation that occurs in the idealized environmental cooling experiments shows a wave-like character, once it reaches the center a relatively steady sustained deep upward motion ensues. The low-level inflow associated with this circulation becomes substantial, reaching magnitudes of approximately $0.8 \mathrm{~m} \mathrm{~s}^{-1}$ in these experiments.

These experiments indicate that the large-scale clear-sky environmental cooling mechanism (Dudhia, 1989; Tao et al., 1996) is also having a noticeable effect. During the early stages of the simulation, before an extensive cloud shield develops aloft, it leads to an increase in humidity that favors the development of moist convection. It is possible that for the case of a prolonged genesis period where convection is sporadic and there are large regions of cloud-free air, this mechanism could be the main driver of diurnal cycles of convective activity in a tropical disturbance. After the formation of a cloud shield the large-scale surrounding environment continues to cool significantly during the nighttime. It is not completely clear from the experiments how influential this aspect is on the cloud system. What stands out from the idealized experiments is the very large impact of differential forcing on relative humidity and potential temperature in the core of the system, so it seems unlikely that the large-scale clear-sky environmental cooling mechanism is as important a factor in influencing the development of a tropical disturbance once an extensive cloud canopy has formed. The simulation with uniform cooling applied throughout the domain supports this conclusion (Experiment 22), since the system did not develop as fast as for the differential forcing case (Experiment 17).
The very large heating and cooling rates that occur at the canopy top and the large heating rate at the stratiform ice base did not seem to have much influence on the genesis rate. Experiment 21 with radiative forcing aloft turned off still underwent genesis almost as quickly as Experiment 13 with radiative forcing aloft. Experiments 16 and 18, with radiative forcing aloft but no environmental forcing, did not develop quickly, again supporting this conclusion. It is however possible that the large variations of heating and cooling at the canopy top could influence the areal extent of cloud cover aloft. This study has not looked at this issue.

The weak mid-level longwave warming that occurs in the core of the system when radiation is included appears to have a fairly small effect on the genesis rate, but not an insignificant one. Experiments 16 and 18 with radiation only in the core both underwent genesis quicker than the no-radiation case, probably because of the mid-level warming. For the simulation with radiative cooling only (Experiment 23), the system underwent genesis considerably slower than for the case with positive and negative radiative forcings (Experiment 13), which is consistent with the view that mid-level warming enhances the genesis rate. The idealized simulation with mid-level warming in the core (Experiment 8 ) showed increased relative humidity aloft, and mid-level radiation warming also clearly contributes to increasing the horizontal differential radiative forcing at night shown in Fig. 24. However, it is not the dominant radiative influence on the genesis rate in these simulations. It is predominantly the nighttime cooling in the environment that is responsible for creating the strong gradient of differential forcing that leads to an increased genesis rate.

The idealized experiments with a prescribed cooling outside a wide core (Experiment 6 ) and the strong vortex simulation (Experiment 10), both suggest that for a large intense tropical cyclone the effects of differential radiative forcing caused by mean environmental cooling at low and mid levels might enhance convection on the periphery of the system. It would be interesting to examine this aspect in future work.

As discussed in the introduction, several previous numerical modeling studies of MCSs concluded that the differential radiative forcing mechanism proposed by Gray and Jacobson (1977) played only a minor role. These results are at variance with the present study, and it is not clear why these previous investigations did not find a significant effect. Gray and Jacobson (1977) stated that the more intense the convection and the more associated it is with an organized weather system, the more evident the diurnal cycle. The system simulated in this study with radiation activated probably has more intense convection than those simulated in the previous studies and it is certainly an organized and persistent system surrounded by a relatively cloud-free environment, so this is likely to be a contributing factor responsible for the different result. Also, the majority of the previous MCS studies were of convective lines with a leading convective region and a predominantly trailing stratiform region, so that the lo- 
cation of the strongest differential radiative forcing may not have coincided with the location of the strongest convection.

This study may have ramifications for why tropical cyclogenesis often occurs in weak vertical wind shear (e.g., Gray, 1968; McBride and Zehr, 1981). Vertically sheared environments with winds aloft significantly different than those at low levels may not be favorable for the formation of symmetrical optically thick stratiform canopies that remain above the center of low-level convergence. This could be a contributing factor for why large vertical wind shear is unfavorable for tropical cyclogenesis.

The validity of this study depends crucially on whether significant differential radiative forcing actually exists between tropical disturbances and their surrounding environment. The physical processes are complex, involving interaction between radiation and microphysics. Moreover, accurate numerical modeling of these processes requires that the grid resolution be good enough to realistically simulate deep convective cells since they are primarily responsible for the formation of an extensive cloud canopy aloft. Further studies are necessary, particularly ones that simulate real systems, to see whether this radiative mechanism is indeed playing a major role.

Acknowledgements. The author is extremely grateful for the help given by Saurabh Barve, who generously volunteered a considerable amount of time to solving several compiling issues with RAMS. Stephen Saleeby provided a new updated version of the RAMS code including the binned scheme for cloud droplet riming. Also, discussions regarding the radiation scheme were very helpful. Norman Wood undertook a valuable comparison between the Harrington scheme used in RAMS and BUGSrad, showing that they gave similar radiative heating profiles for the Jordan sounding. Furthermore, he pointed out the sensitivity of the cooling rates aloft to small amounts of water vapor in the upper troposphere. The author is grateful to Donavan Wheeler for aid with the data analysis code, and to Roger Pielke Sr. and Dallas Staley for helpful discussions. This work was supported in part by the National Science Foundation, under grants NSF AGS 0965721, NSF AGS 1445875 and NSF AGS 1415244. Joan M. Nicholls also contributed significantly to supporting this project.

Edited by: T. J. Dunkerton

\section{References}

Adams-Selin, R. D. and Johnson, R. H.: Mesoscale surface pressure and temperature features associated with bow echoes, Mon. Wea. Rev., 138, 212-227, 2010.

Adams-Selin, R. D. and Johnson, R. H.: Examination of gravity waves associated with the 13 March 2003 bow echo, Mon. Wea. Rev., 141, 3735-3756, 2013.

Bell, M. M.: Air-sea enthalpy and momentum exchange at major hurricane wind speeds. Ph. D. thesis, Naval Post Graduate School, Monterey, California, 2012.
Bretherton, C. S. and Smolarkiewicz, P. K.: Gravity waves, compensating subsidence and detrainment around cumulus clouds, J. Atmos. Sci., 46, 740-759, 1989.

Browner, S. P., Woodley, W. L., and Griffith, C. G.: Diurnal oscillation of the area of cloudiness associated with tropical storms, Mon. Wea. Rev., 105, 856-864, 1977.

Bu, Y. P., Fovell, R. G., and Corbosiero, K. L.: Influence of cloudradiative forcing on tropical cyclone structure, J. Atmos. Sci., 71, 1644-1662, 2014.

Bryan, G. H. and Parker, M. D.: Observations of a squall line and its near environment using high-frequency rawinsonde launches during VORTEX2, Mon. Wea. Rev., 138, 4076-4097, 2010.

Clark, T. L. and Farley, R. D.: Severe downslope windstorm calculations in two and three spatial dimensions using anelastic grid nesting: A possible mechanism for gustiness, J. Atmos. Sci., 41, 329-350, 1984.

Cotton, W. R., Pielke Sr., R. A., Walko, R. L., Liston, G. E., Tremback, C. T., Jiang H., McAnelly, R. L., Harrington, J. Y., and Nicholls, M. E.: RAMS 2001: Current status and future directions, Meteor. Atmos. Phys, 82, 5-29, 2003.

Craig, G.: Numerical experiments on radiation and tropical cyclones. Quart. J. Roy. Meteor. Soc., 122, 415-422, 1996.

Davis, C. A. and Ahijevych, D. A.: Mesoscale structural evolution of three tropical weather systems observed during PREDICT, J. Atmos. Sci., 69, 1284-1305, 2012.

Dudhia, J.: Numerical Study of Convection Observed during the Winter Monsoon Experiment Using a Mesoscale TwoDimensional Model, J. Atmos. Sci., 46, 3077-3107, 1989.

Dunkerton, T. J., Montgomery, M. T., and Wang, Z.: Tropical cyclogenesis in a tropical wave critical layer: easterly waves, Atmos. Chem. Phys., 9, 5587-5646, doi:10.5194/acp-9-5587-2009, 2009.

Eliassen, A.: Slow thermally or frictionally controlled meridional circulation in a circular vortex, Astrophys. Norv., 5, 19-60, 1951.

Fovell, R. G.: Upstream influence of numerically simulated squallline storms, Quart. J. Roy. Meteor. Soc., 128, 893-912, 2002.

Fovell, R. G., Mullendore, G. L., and Kim, S.-H.: Discrete propagation in numerically simulated nocturnal squall lines, Mon. Wea. Rev., 134, 3735-3752, 2006.

$\mathrm{Fu}$, Q., Krueger, S. K., and Liou, K. N.: Interactions of radiation and convection in simulated tropical cloud clusters, J. Atmos. Sci., 52, 1310-1328, 1995.

Gray, W. M.: Global view of the origin of tropical disturbances and storms, Mon. Wea. Rev., 96, 669-700, 1968.

Gray, W. M. and Jacobson, R. W. J.: Diurnal variation of deep cumulus convection, Mon. Wea. Rev., 105, 1171-1188, 1977.

Hack, J. J. and Schubert, W. H.: The role of convective-scale processes in tropical cyclone development, Ph.D. thesis, Colorado State University, Ft. Collins, CO., 1980.

Haertel, P. T. and Johnson, R. H.: The linear dynamics of squall line mesohighs and wake lows, J. Atmos. Sci., 57, 93-107, 2000.

Harrington, J. Y.: The effects of radiative and microphysical processes on simulated warm and transition season arctic Stratus. Ph.D. thesis, Colorado State University, Ft. Collins, CO., 298 pp,. 1997.

Harrington, J. Y., Reisin, T., Cotton, W. R., and Kreidenweis, S. M.: Exploratory cloud resolving simulations of arctic stratus. Part II: Transition-season clouds, Atmos. Res., 51, 45-75, 1999. 
Hendricks, E. A. and Montgomery, M. T.: The role of "vortical" hot towers in the formation of Tropical Cyclone Diana (1984), J. Atmos. Sci., 61, 1209-1231, 2004.

Hill, G. E.: Factors controlling the size and spacing of cumulus clouds as revealed by numerical experiments, J. Atmos. Sci., 31, 646-673, 1974.

Hobgood, J. S.: A possible mechanism for the diurnal oscillation of tropical cyclones, J. Atmos. Sci., 43, 2901-2922, 1986.

James, R. P. and Markowski, P. M.: A numerical investigation oft he effects of dry air aloft on deep convection, Mon. Wea. Rev., 138, 140-161, 2009.

Jordan, C. L.: Mean soundings for the West Indies area, J. Meteor., 15, 91-97, 1958.

Kilroy, G. and Smith R. K.: A numerical study of rotating convection during tropical Cyclogenesis, Quart. J. Roy. Meteor. Soc., 139, 1255-1269, 2013.

Klemp, J. B. and Wilhelmson, R. B.: The simulation of threedimensional convective storm dynamics, J. Atmos. Sci., 35, 1070-1086, 1978.

Kossin, J. P.: Daily hurricane variability inferred from GOES infrared imagery, Mon. Wea. Rev., 130, 2260-2270, 2002.

Lajoie, F. A. and Butterworth I. J.: Oscillation of high-level cirrus and heavy precipitation around Australian region tropical cyclones, Mon. Wea. Rev., 112, 535-544, 1984.

Lane, T. P. and Reeder, M. J.: Convectively-generated gravity waves and their effect on the cloud environment, J. Atmos. Sci., 58, 2427-2440, 2001.

Lane, T. P. and Zhang, F.: Coupling between Gravity Waves and Tropical Convection at Mesoscales, J. Atmos. Sci., 68, 25822598, 2011.

Lilly, D. K.: On the numerical simulation of buoyant convection, Tellus, 14, 148-172, 1962.

Lin, Y. L. and Smith, R. B.: Transient dynamics of airflow near a local heat source, J. Atmos. Sci., 43, 40-49, 1986.

Liu, C. and Moncrieff, M. W.: Effects of convectively generated gravity waves and rotation on the organization of convection, J. Atmos. Sci., 61, 2218-2227, 2004.

Mapes, B. E.: Gregarious tropical convection, J. Atmos. Sci., 50, 2026-2037, 1993.

McAnelly, R. L., Nachamkin, J. E., Cotton, W. R., and Nicholls, M. E.: Upscale evolution of MCSs: Doppler radar analysis and analytical investigation, Mon. Wea.Rev., 125, 1083-1110, 1997.

McBride, J. L. and Zehr, R.: Observational analysis of tropical cyclone formation - Part II: Comparison of non-developing versus developing systems, J. Atmos. Sci., 38, 1132-1151, 1981.

Melhauser, C. and Zhang, F.: Diurnal radiation impact on the pregenesis environment of Hurricane Karl (2010), J. Atmos. Sci., 71, 1241-1259, 2014.

Meyers, M. P., Walko, R. L., Harrington, J. Y., and Cotton, W. R.: New RAMS cloud microphysics parameterization. Part II: The two-moment scheme, Atmos. Res., 45, 3-39, 1997.

Miller, R. A. and Frank, W. M.: Radiative forcing of simulated tropical cloud clusters, Mon. Wea. Rev., 121, 482-498, 1993.

Mitchell, D. L., Macke, A., and Liu, Y.: Modeling cirrus clouds: Part II. Treatment of radiative properties, J. Atmos. Sci., 53, 29672988, 1996.

Montgomery, M. T., Nicholls, M. E., Cram, T. A., and Saunders, A. B.: A vortical hot tower route to tropical cyclogenesis, J. Atmos. Sci., 63, 355-386, 2006.
Montgomery, M. T., Wang, Z., and Dunkerton, T. J.: Coarse, intermediate and high resolution numerical simulations of the transition of a tropical wave critical layer to a tropical storm, Atmos. Chem. Phys., 10, 10803-10827, doi:10.5194/acp-1010803-2010, 2010.

Muramatsu, T.: Diurnal variations of satellite-measured $\mathrm{T}_{B B}$ areal distribution and eye diameter of mature typhoons, J. Meteor. Soc. Japan, 61, 77-89, 1983.

Nicholls, M. E.: A comparison of the results of a two-dimensional numerical simulation of a tropical squall line with observations, Mon. Wea. Rev., 115, 3055-3077, 1987.

Nicholls, M. E. and Pielke Sr., R. A.: Thermally-induced compression waves and gravity waves generated by convective storms, J. Atmos. Sci., 57, 3251-3271, 2000.

Nicholls, M. E. and Montgomery, M. T.: An examination of two pathways to tropical cyclogenesis occurring in idealized simulations with a cloud-resolving numerical model, Atmos. Chem. Phys., 13, 5999-6022, doi:10.5194/acp-13-5999-2013, 2013.

Nicholls, M. E., Pielke Sr., R. A., and Cotton, W. R.: Thermally forced gravity waves in an atmosphere at rest, J. Atmos. Sci., 48, 1869-1884, 1991.

Nolan, D. S.: What is the trigger for tropical cyclogenesis?, Aust. Met. Mag. 56, 241-266, 2007.

Pandya, R. E. and Durran, D. R.: The influence of convectively generated thermal forcing on the mesoscale circulation around squall lines, J. Atmos. Sci., 53, 2924-2951, 1996.

Pandya, R. E, Durran, D. R., and Bretherton, C.: Comments on "Thermally forced gravity waves in an atmosphere at rest", J. Atmos. Sci., 50, 4097-4101, 1993.

Pandya, R. E., Durran, D. R., and Weisman, M. L.: The influence of convective thermal forcing on the mesoscle circulaton around three-dimensional squall lines, J. Atmos. Sci., 57, 29-45, 2000.

Pendergrass, A. G. and Willoughby, H. E.: Diabatically induced secondary flows in tropical cyclones - Part I: Quasi-steady forcing, Mon. Wea, Rev., 137, 805-821, 2009.

Pielke Sr., R. A., Cotton, W. R., Walko, R. L., Tremback, C. J., Lyons, W. A., Grasso, L. D., Nicholls, M. E., Moran, M. D., Wesley, D. A., Lee, T. J., and Copeland, J. H.: A comprehensive meteorological modeling system - RAMS, Meteorol. Atmos. Phys., 49, 69-91, 1992.

Raymond, D. J.: Prescribed heating of a stratified atmosphere as a model for moist convection, J. Atmos. Sci., 43, 1101-1111, 1986.

Ritter, B. and Geleyn, J.-F.: A comprehensive radiation scheme for numerical weather prediction models with potential applications in climate simulations, Mon. Wea. Rev., 120, 303-325, 1992.

Saleeby, S. M. and Cotton, W. R.: A binned approach to clouddroplet riming implemented in a bulk microphysics model, J. Appl. Meteorol. Climatol., 47, 694-703, 2008.

Shige, S. and Satomura, T.: The gravity wave response in the troposphere around deep convection, J. Meteor. Soc. Japan, 78, 789801, 2000.

Shu, H.-L., Zhang, Q.-H., and Xu, B.: Diurnal variation of tropical cyclone rainfall in the western north Pacific in 2008-2010, Atmos. Oceanic Sci. Lett., 6, 103-108, 2013.

Smagorinsky, J. S.: General circulation experiments with the primitive equations, 1: The basic experiment, Mon. Wea. Rev., 91, 99-164, 1963. 
Steranka, J., Rodgers, E. B., and Gentry, R. C.: The diurnal variation of Atlantic ocean tropical cyclone cloud distribution inferred from geostationary satellite infrared measurements, Mon. Wea. Rev., 112, 2338-2344, 1984.

Sundqvist, H.: Numerical simulation of the development of tropical cyclones with a ten-level model - Part II., Tellus, 22, 504-510, 1970.

Tao, W.-K., Simpson, J., Lang, S., Sui, C.-H., Ferrier, B., and Chou, M.-D.: Mechanisms of cloud-radiation interaction in the tropics and midlatitudes, J. Atmos. Sci. 53, 2624-2651, 1996.

Tripoli, G. J. and Cotton, W. R.: The use of ice-liquid water potential temperature as a thermodynamic variable in deep atmospheric models, Mon. Wea. Rev., 109, 1094-1102, 1981.

Tulich, S. N. and Mapes, B. E.: Multiscale convective wave disturbances in the tropics: Insights from a two-dimensional cloudresolving model, J. Atmos. Sci., 65, 140-155, 2008.

Vigh, J. L. and Schubert, W. H.: Rapid development of the tropical cyclone warm core, J. Atmos. Sci., 66, 3335-3350, 2009.

Walko, R. L., Cotton, W. R., Harrington, J. L., Meyers, M. P.: New RAMS cloud microphysics parameterization - Part I: The singlemoment scheme, Atmos. Res., 38, 29-62, 1995.
Wang, Z.: Thermodynamic aspects of tropical cyclone formation, J. Atmos. Sci., 69, 2433-2451, 2012.

Wang, Z., Montgomery, M. T., and Dunkerton, T. J.: Genesis of PreHurricane Felix (2007). Part I: The role of the wave critical layer, J. Atmos. Sci., 67, 1730-1744, 2010

Webster, P. J. and Stephens, G. L.: Tropical upper-tropospheric extended clouds: Inference from winter MONEX, J. Atmos. Sci., 37, 1521-1541, 1980.

Wirth, V. and Dunkerton, T. J.: A unified perspective on the dynamics of axisymmetric hurricanes and monsoons, J. Atmos. Sci., 63, 2529-2547, 2006

Wirth, V. and Dunkerton, T. J.: The dynamics of eye formation and maintenance in axisymmetric diabatic vortices, J. Atmos. Sci., 66, 3601-3620, 2009.

$\mathrm{Xu}$, K.-M. and Randall, D. A.: Impact of interactive radiative transfer on the macroscopic behavior of cumulus ensembles. Part II: Mechanisms for cloud-radiation interactions, J. Atmos. Sci., 52, 800-817, 1995.

Zhang, C. and Chou, M.-D.: Variability of Water Vapor, Infrared Radiative Cooling, and Atmospheric Instability for Deep Convection in the Equatorial Western Pacific, J. Atmos. Sci., 56, 711-723, 1999. 